\title{
Identification of touch neurons underlying dopaminergic pleasurable touch and sexual receptivity
}

Leah Middleton ${ }^{1,2}$, Melanie Schaffler ${ }^{1,2}$, Isabella Succi ${ }^{1}$, William Foster ${ }^{1}$, Mark Gradwell ${ }^{3,4}$, Manon Bohic ${ }^{3,4}$, Lindsay Ejoh ${ }^{2}$, Victoria Abraira ${ }^{3,4}$, Ishmail Abdus-Saboor ${ }^{1 *}$

${ }^{1}$ Zuckerman Mind Brain Behavior Institute and Department of Biological Sciences, Columbia University, New York, New York, United States of America

${ }^{2}$ Neuroscience Graduate Group, University of Pennsylvania, Philadelphia, PA, USA

${ }^{3}$ Cell Biology and Neuroscience Department, Rutgers University, The State University of New Jersey, New Brunswick, United States of America

${ }^{4}$ W.M. Keck Center for Collaborative Neuroscience, Rutgers University, The State University of New Jersey, New Brunswick, United States of America

"Corresponding author: ia2458@columbia.edu

\begin{abstract}
Pleasurable touch during social behavior is the key to building familial bonds and meaningful connections. One form of social touch occurs during sex. Although sexual behavior is initiated in part by touch, and touch is ongoing throughout copulation, the identity and role of sensory neurons that transduce sexual touch remain unknown. A population of sensory neurons labeled by the G-protein coupled receptor Mrgprb4 detect stroking touch in mice ${ }^{1,2}$. Here, we study the social relevance of this population by genetically engineering mice to allow activation or ablation of Mrgprb4-lineage neurons and reveal that these neurons are required for sexual receptivity and sufficient to activate reward circuitry. Even in social isolation, optogenetic stimulation of Mrgprb4-lineage neurons through the back skin is sufficient to induce a conditioned place preference and a striking dorsoflexion resembling the lordotic copulatory posture in females. In the absence of Mrgprb4-lineage neurons, female mice no longer find male mounts rewarding: sexual receptivity is supplanted by aggression and a coincident decline in dopaminergic release in the mesolimbic reward pathway. In addition to sexual behavior, Mrgprb4lineage neurons are also required for social postures induced by female-to-female back touch. Together, these findings establish that Mrgprb4-lineage neurons are the first neurons of a skin-to-brain circuit encoding the rewarding quality of social touch.
\end{abstract}




\section{Introduction}

The pleasure of a partner's caress or a child's embrace begins with mechanical signals transduced by neurons in our skin. The way our brain interprets these instances of social touch is critical for our well-being. Despite the centrality of socially rewarding touch in our daily lives, the neurons in the skin that detect social touch and shape the valence of perception remain unknown. This gap in knowledge is critical, especially when considering the nature of neurodevelopmental disorders like autism spectrum disorder, where gentle touch and socially rewarding behaviors are aversive ${ }^{3-5}$.

A class of sensory neurons in humans that are linked to gentle stroking are termed C-tactile afferents ${ }^{6,7}$, and there are putative populations of these neurons in the mouse ${ }^{8-}$ 10. One such class of neurons in mice express the G-protein coupled receptor Mrgprb4 and share anatomical and physiological similarities with human C-tactile afferents ${ }^{1,2}$. These hairy skin-innervating C-fibers respond to gentle stroking and produce a conditioned place preference, suggesting their activation is rewarding ${ }^{1,2}$. Since the Mrgprb4+ touch neurons fit the description of cells in the skin that might promote ethologically relevant rewarding touch, we used a combination of mouse genetics, novel behavioral paradigms, and in vivo brain imaging to connect the skin and brain by dissecting the role of Mrgprb4+ neurons in socially rewarding behaviors.

\section{Results}

Focalized activation of Mrgprb4-lineage neurons in the skin is rewarding and induces lordosis-like posture in female mice

We reasoned that focal activation of Mrgprb4+ neurons in the back skin might mimic touch among mice, so we used the blue light sensitive ion channel channelrhodopsin (ChR2) to focally stimulate Mrgprb4+ neurons in vivo. To accomplish this goal, we used an Mrgprb4 ${ }^{\text {Cre }}$ driver that functions as a lineage tracer to express a ChR2-eYFP fusion protein in Mrgprb4-lineage neurons (Fig 1a). We confirmed this genetic targeting strategy with RNA Scope in situ hybridization and immunostaining (Fig 1b,c). Because Mrgprb4 expression begins P4-5 in a population of progenitors broader than the Mrgprb4-expressing population of adult neurons ${ }^{11}$, we characterized the expression of ChR2-eYFP in populations of dorsal root ganglion neurons known to share lineage with Mrgprb4+ neurons. Using RNA Scope in situ hybridization, we characterized co-expression of ChR2-eYFP with Mrgprb4, Mrgprd, Mrgprc11, MrgprA3, TrpA1, TrpV1, and tyrosine hydroxylase (TH) (Supplemental Figure 1). We found the expression of ChR2 across these populations consistent with the expected developmental expression of Mrgprb4 ${ }^{11}$ (Fig 1d,e). ChR2 was co-expressed in 93.6\% of adult Mrgprb4+ neurons; $35.2 \%$ of TrpA1+ neurons; $36.2 \%$ of Mrgprd+ neurons; $46.8 \%$ of Mrgprc11+ neurons; and $61.3 \%$ of Mrgpra3+ neurons (Fig 1d). Notably, there was little to no overlap between ChR2 (Mrgprb4-lineage expression) with $\mathrm{TH}$, which is a marker of other C-LTMRs ${ }^{12}$, confirming that the Mrgprb4 lineage neurons are a distinct population of C-fibers. With confirmation of our genetic targeting strategy to activate Mrgprb4-lineage neurons with 

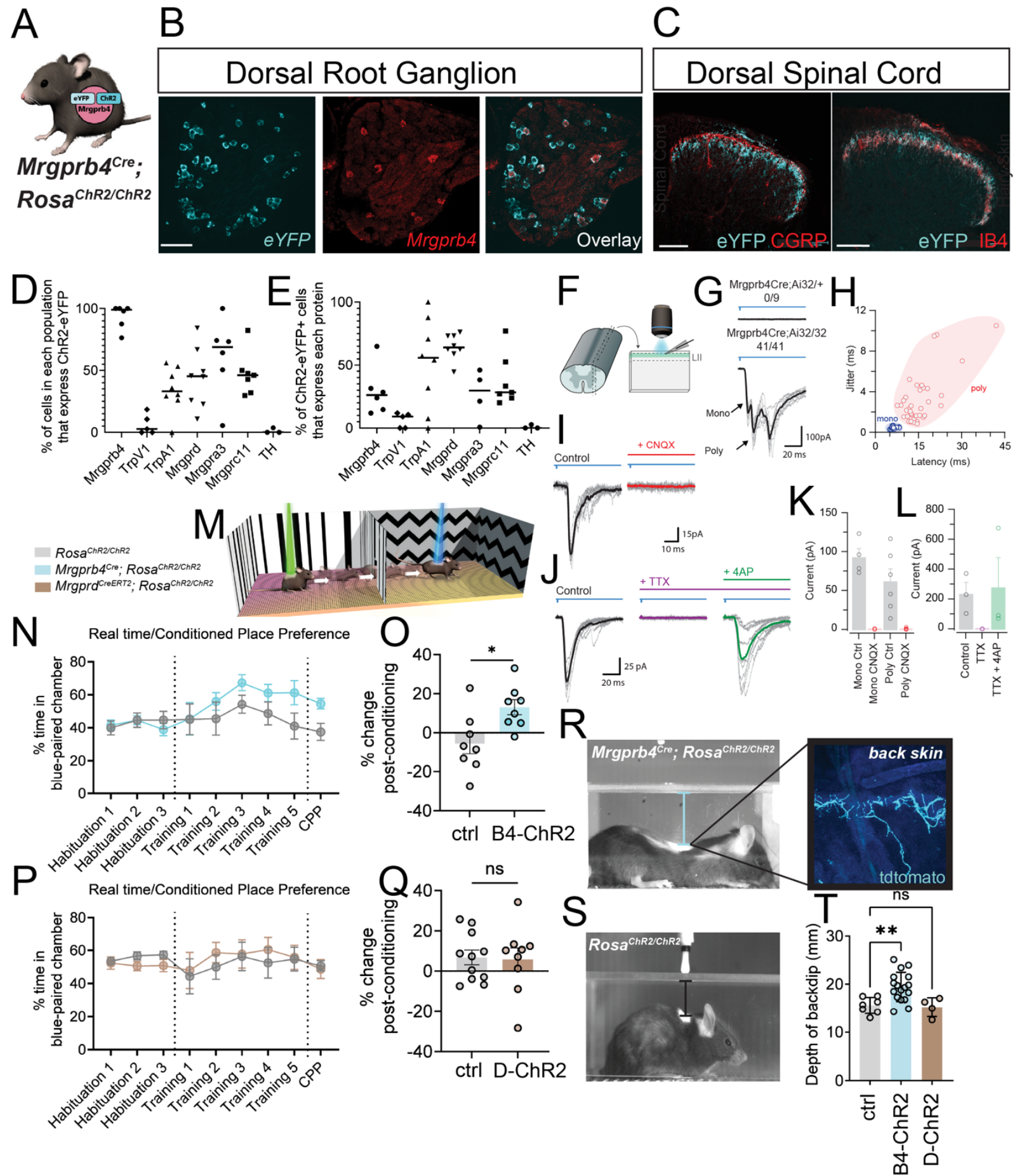

ChR2, we next asked if we could induce light-evoked, synaptically driven currents to second-order neurons downstream in the dorsal horn of the spinal cord by combining optogenetics with whole cell patch clamp physiology (Fig 1f). Interestingly, recording from LII of the spinal cord of a Mrgprb4 ${ }^{\mathrm{Cre}} ; \mathrm{Rosa}^{\mathrm{ChR} 2 /+}$ heterozygous mice yielded no lightinduced currents, while all recordings from Mrgprb4Cre;RosaChR2/ChR2 homozygotes exhibited mono- and/or poly-synaptic inputs (Fig 1g,h; Supplemental Figure 2). 15/39 neurons recorded fired action potentials following $1 \mathrm{~ms}$ photostimulation. Monosynaptic 
Figure 1: Focalized activation of Mrgprb4-lineage neurons in the back skin is rewarding and induces a lordosislike posture in female mice. A) Mrgprb4 ${ }^{\text {Cre }}$ mice express ChR2-eYFP in a Cre-dependent manner. B) RNA Scope in situ hybridization in DRG to quantify the expression of ChR2 in Mrgprb4+ cells. Scale bars represent $100 \mu \mathrm{m}$. C) Immunofluorescence staining in spinal cord dorsal horn. Mrgprb4+ terminals innervate lamina II, inferior to CGRP+ terminals in lamina I and overlapping with IB4+ terminals in lamina II. Scale bars represent $100 \mu \mathrm{m}$. D) Expression of eYFP in different populations of DRG neurons that share lineage with Mrgprb4. From RNA Scope in situ hybridization in supplemental figure 1. E) Expression of different lineage populations among ChR2-eYFP+ cells. See supplemental figure 1 for original RNA Scope in situ hybridization images. F) Schematic illustrating spinal cord slice electrophysiological recordings from lamina II during optogenetic stimulation of terminals. G) Mono- or poly-synaptic light induced currents only in Mrgprb4 ${ }^{\text {Cre; }}$ RosaChR2/ChR2 mice. H) Quantification of light induced currents from Mrgprb4 ${ }^{C r e}$; Rosa ${ }^{C h R 2 / C h R 2}$ mice with jitter used to determine mono- or poly-synaptic transmission. I) Light-induced currents in Mrgprb4 Mrgprb4 ${ }^{C r e}$; Rosa ${ }^{C h R 2 / C h R 2}$ mice with TTX, but not in the presence of TTX + 4AP. K,L) Quantification of data presented in I,J. M) Schematic illustrating our real time/conditioned place preference assay. N) Mrgprb4 ${ }^{\text {Cre }}$; RosaChR2/ChR2 females gradually spend more time in the blue laser chamber compared to green laser chamber during the training days (lasers on) and O) spend significantly more time in the chamber they learned to associate with blue light on the test day (lasers off) compared to habituation days, ( ${ }^{*} P<0.05$, unpaired t-test.) P,Q) Mrgprd ${ }^{C R E-E R T 2}$; RosaChR2/ChR2 females do not develop a preference for the blue laser-paired chamber. R,S) Stills from high speed videography to closely examine the behavior during the preferable transdermal optogenetic activation of Mrgprb4-lineage neurons. R) On the right, immunofluorescence staining of Cre-tdtomato+ terminals in the hairy skin. Mrgprb4Cre; RosaChR2/ChR2 females (R) exhibit a striking lordosis-like dorsiflexion in response to Mrgprb4-lineage neuron activation, a behavior absent from (S) $R_{0 s a}{ }^{C h R 2 / C h R 2}$ female littermates. T) This posture is quantified as the back's maximum distance from the chamber ceiling over the course of 20s optogenetic stimulation. Mrgprb4 ${ }^{C r e} ;$ RosaChR2/ChR2 females exhibit significantly greater backdip than the spontaneous movements of Rosa ${ }^{C h R 2 / C h R 2}$ littermates ( ${ }^{* *} P<0.01$ ), while MrgprdCRE-ERT2; RosaChR2/ChR2 females do not (One-way ANOVA).

nature of inputs was confirmed with application of TTX+4AP, and both mono and polysynaptic Mrgprb4 inputs to second order neurons can be abolished by application of CNQX, demonstrating both sodium channel-dependent firing of Mrgprb4-lineage neurons and glutamatergic synaptic transmission between Mrgprb4-lineage neurons and secondorder neurons (Fig 1i-I). Together, the genetic targeting and electrophysiological recordings demonstrate the feasibility of optogenetically activating Mrgprb4-lineage neurons to evoke behavior.

Previous work demonstrated that chemogenetic activation of Mrgprb4-lineage neurons in juvenile males induces a conditioned place preference ${ }^{2}$, suggesting activation of these neurons is inherently pleasant or rewarding. To test whether focal activation of these afferents in the back skin is similarly preferable, we developed a conditioned place preference assay in which each chamber was paired with laser light administered transdermally through the shaved back skin. We used Mrgprb4 ${ }^{C r e} ;$ RosaChR2/ChR2 mice given our result with spinal cord slice physiology (Fig 1f,g). One chamber had ChR2activating blue light, and the had other non-stimulating green light as a control (Fig $1 \mathrm{~m})$. Mrgprb4Cre; RosaChR2/ChR2 females had a significant preference for the chamber associated with blue light stimulation after training compared to Cre negative littermates, suggesting that focal activation of Mrgprb4-lineage neurons in the back skin is inherently pleasant or rewarding (Fig 1n,o). To confirm the specificity of this result to the Mrgprb4lineage population, we performed analogous experiments with Mrgprd ${ }^{\text {Cre-ERT2; }}$ RosaChR2/ChR2 mice, as Mrgprb4 and Mrgprd mark related sensory neuron populations that share developmental lineage ${ }^{13}$. We and others have previously demonstrated that optogenetic activation of Mrgprd+ neurons is neutral at baseline conditions ${ }^{14-16}$, and we 
recapitulated those findings here in our newly developed optogenetic placed preference paradigm showing no preference for blue light when Mrgprd+ neurons are activated through the back skin (Fig 1p,q). Thus, the positive valence is uniquely associated with activation of Mrgprb4-lineage neurons. Focal activation in the back is sufficient to drive this preference, suggesting the Mrgprb4-lineage neurons may detect pleasant tactile contact to the back skin.

Behaviors that result from optogenetic activation of sensory neurons that sense pain or itch are intuitive to interpret. For example, we and others have shown that selective optogenetic activation of nociceptive neurons in mice evokes pain behaviors, such as paw withdrawal, licking, and shaking, while activation of itch-sensing neurons evokes stereotyped itch behaviors, such as scratching ${ }^{13,14,17-21}$. To uncover a stereotyped behavior evoked by optogenetic activation of Mrgprb4-lineage neurons in freely behaving mice, we combined optogenetic stimulation with high-speed videography at 750 frames per second to capture behavior at high spatial and temporal resolution. Intriguingly, a lordosis-like posture, involving robust dorsiflexion, appeared as a stereotyped response to selective activation of Mrgprb4-lineage neurons in the back skin (Fig 1r). We quantified this posture as the maximum distance the back concaved from the ceiling of the plastic chamber over the course of 20 s of optogenetic stimulation (Fig 1t). The posture is not observed upon optogenetic activation in the same experimental chambers of either Mrgprd+ neurons (Fig 1t) or Mrgpra3+ neurons (Supplemental Figure 3), two related populations of neurons that share lineage with Mrgrprb4. Thus, this posture is specific to activation of Mrgprb4-lineage neurons and represents the first stereotyped behavioral response to the selective activation of pleasant touch. Because this response resembles the sexual receptivity lordosis posture, and occurred in female mice with optogenetic stimulation directed to the back, we hypothesized that Mrgprb4-lineage neurons might play a role in sexual receptivity and other touch-dependent socially rewarding behaviors.

Mrgprb4-lineage neurons are required for sexual receptivity and female-female social postures but not social interest

Next, we interrogated the role of Mrgprb4 neurons in two natural behaviors where female rodents display prominent dorsiflexion postures in response to touch: 1) lordosis - the female sexually receptive posture which includes dorsiflexion in response to male tactile input to the back and flanks ${ }^{22-26}$ and 2) crawling underneath cage mates which involves a dorsiflexion of the spine in response to the cage mate on top, and has recently been associated with oxytocin neuron activation in rats ${ }^{27}$. We refer to this behavior as the "conspecific crawl." We focused on female social behaviors because lordosis is a female behavior and conspecific tactile input may be conflated with aggression or dominance behavior in males. 
To determine whether Mrgprb4-lineage neurons are required for either of these touch-dependent social behaviors, we ablated the population using a Cre-dependent diphtheria toxin mediated ablation (Fig 2a; lineage quantification in Fig 1d,e). We used RNA Scope in situ hybridization to confirm the elimination of dorsal root ganglion (DRG) cell bodies that express Mrgprb4 (Fig 2b). Mrgprb4Cre; Rosa ${ }^{D T A}$ mice did not exhibit any motor abnormalities that might conflate interpretations of our results (Fig 2c,d). To determine if the Mrgprb4-lineage neurons are required in females to detect male mounts and facilitate lordosis, we conducted a lordosis quotient assay ${ }^{22,28}$. To control for natural fluctuations in the estrous cycle, we ovariectomized females (OVX) and subsequently treated them with both estradiol and progesterone to mimic a state of behavioral estrus at the time of the assay, as previously described ${ }^{29}$ (Fig 2e). Mrgprb4 ${ }^{\text {Cre; }}$ Rosa ${ }^{\text {DTA }}$ females and cage mate controls were paired with stud males that had recently fathered multiple litters, confirming their sexual competency. We measured the fraction of receptive responses to total male mounts (lordosis quotient) as well as the average duration the female maintained a receptive posture (sexual receptivity). A female's response was

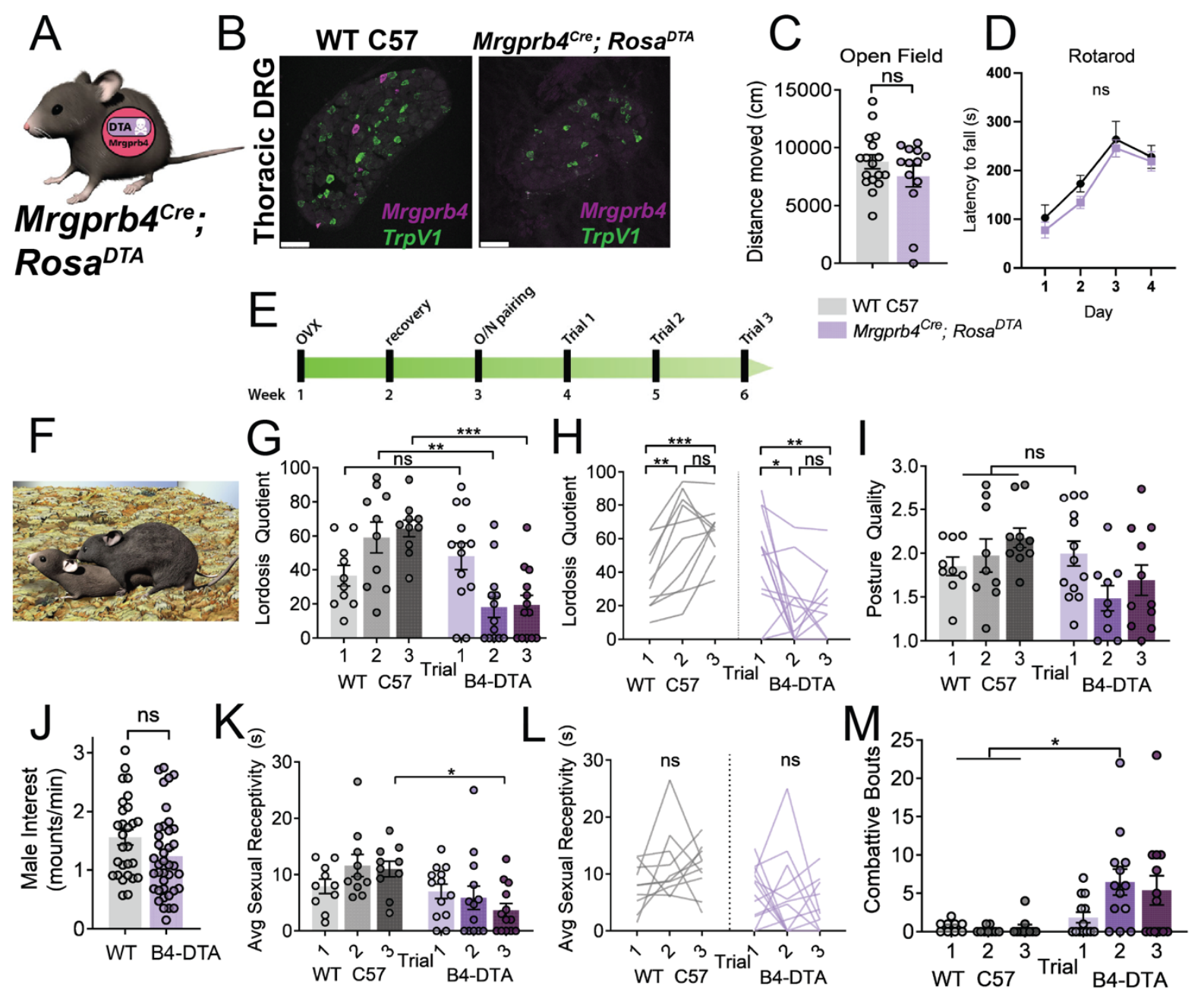


Figure 2: Mrgprb4-lineage neurons are required for female sexual receptivity. A) Graphic representing Mrgprb4 ${ }^{C r e}$; $R_{\text {Rosa }}{ }^{D T A}$ mouseline, expressing DTA in a Cre-dependent manner to ablate Mrgprb4-lineage neurons. B) RNA Scope in situ hybridization of DRGs from B) WT C57 control and C) Mrgprb4 ${ }^{\text {Cre }}$; Rosa ${ }^{D T A}$ female, cell bodies expressing Mrgprb4 (magenta) are absent upon DTA expression while TrpV1 (green) cells remain intact. Scale bars $=75 \mu \mathrm{m}$. C,D) Mrgprb4 ${ }^{\text {Cre }}$; Rosa ${ }^{D T A}$ mice exhibit no motor deficits in open field (unpaired t-test) or rotarod assays (2-way ANOVA). E) Timeline for assessing sexual receptivity with lordosis quotient (LQ) assay: all females are given two weeks to recover after ovariectomy, at which point estradiol and progesterone are administered to put them in behavioral estrus for an overnight pairing with a male. Hormones replaced prior to each LQ trial. F) Assays conducted in the male home cage in the dark cycle. Graphic depicts a sexually receptive female lordosis posture $\mathrm{G}$ ) Lordosis quotient scores for 3 sequential trials for WT C57 and Mrgprb4 ${ }^{C r e}$; Rosa ${ }^{D T A}$ females. ( ${ }^{\star} P<0.05,{ }^{* *} P<0.01,{ }^{* \star *} P<0.001$, One-Way ANOVA). H) Changes in individual mice across the three trials. ( ${ }^{*} P<0.05,{ }^{* *} P<0.01,{ }^{* *} P<0.001$, Repeated Measures ANOVA). I) Posture quality assessed on a scale from 1-3, where 1 is the minimum receptive posture and 3 is the most robust posture (details in methods). J) Male mounting frequency was no different between Mrgprb4 ${ }^{\text {Cre }}$; Rosa ${ }^{D T A}$ females and controls. K) Average sexual receptivity, or duration maintained a receptive posture. ( ${ }^{*} P<0.05$, One way ANOVA). L) Changes in sexual receptivity in individual mice across trials (Repeated measures ANOVA). M) Total number of combative bouts observed for each trial. By the third trial, Mrgprb4 ${ }^{C r e}$; Rosa ${ }^{D T A}$ females exhibited significantly more combative bouts than control females on any trial ( ${ }^{*} P<0.05$, One way ANOVA).

considered receptive if she had all four limbs on the floor of the cage and displayed no attempts to escape. Mrgprb4 ${ }^{\text {Cre }}$; Rosa ${ }^{D T A}$ females had similar lordosis quotient and sexual receptivity to controls on the first week of testing, but, strikingly, their receptivity plummeted on the second pairing, and remained minimal for the third pairing (Fig $2 \mathrm{~g}, \mathrm{~h}, \mathrm{k}, \mathrm{l})$. On the other hand, control females increased in receptivity with each trial, suggesting that intact female mice might be learning to engage in this rewarding behavior (Fig 2g,h).

To determine whether Mrgprb4Cre; RosaDTA females resisted mounts on subsequent pairings because they were incapable of producing a quality lordosis posture, we examined the quality of the posture in each of the three weeks. We found that their posture quality was normal on the first trial, suggesting that Mrgprb4 ${ }^{C r e} ; R_{0 s a^{D T A}}$ females are indeed capable of producing a quality lordosis posture, but choosing not to display lordosis on subsequent trials (Fig 2i). Despite the reduced sexual receptivity of females, males demonstrated equal vigor in mounting in both Mrgprb4Cre; Rosa ${ }^{\text {DTA }}$ females and WT C57 controls (Fig 2j). Concomitant with the sharp decline in sexual receptivity in Mrgprb4 ${ }^{C r e}$; Rosa ${ }^{\text {DTA }}$ females, these females now displayed combative behavior towards the male attempts to mount (Fig $2 \mathrm{~m}$ ). This phenotype is striking, as female mice primed for behavioral estrus are not naturally aggressive ${ }^{29,30}$. Mrgprb4-lineage neurons are therefore not required for a motor output of lordotic dorsiflexion, but must encode a sensation that is necessary for the rewarding nature of sexual touch.

The "conspecific crawl" behavior, in which one female adopts a dorsiflexion posture as she crawls beneath a cage mate (thereby receiving social back contact) (Fig 3a), reminded us of postures we observed with optogenetic stimulation of Mrgprb4lineage neurons (Fig $3 \mathrm{~b}$ ). The resemblance between natural and optogenetically evoked postures led us to ask if Mrgprb4-lineage neurons are required for female-to-female social behavior that involves back touch. We found that when Mrgprb4 ${ }^{C r e}$; Rosa ${ }^{D T A}$ adult females and WT-C57 cage mates were observed for spontaneous social interaction, there was a specific deficit in social touch-induced dorsiflexion, or conspecific crawl (Fig 3e). The 

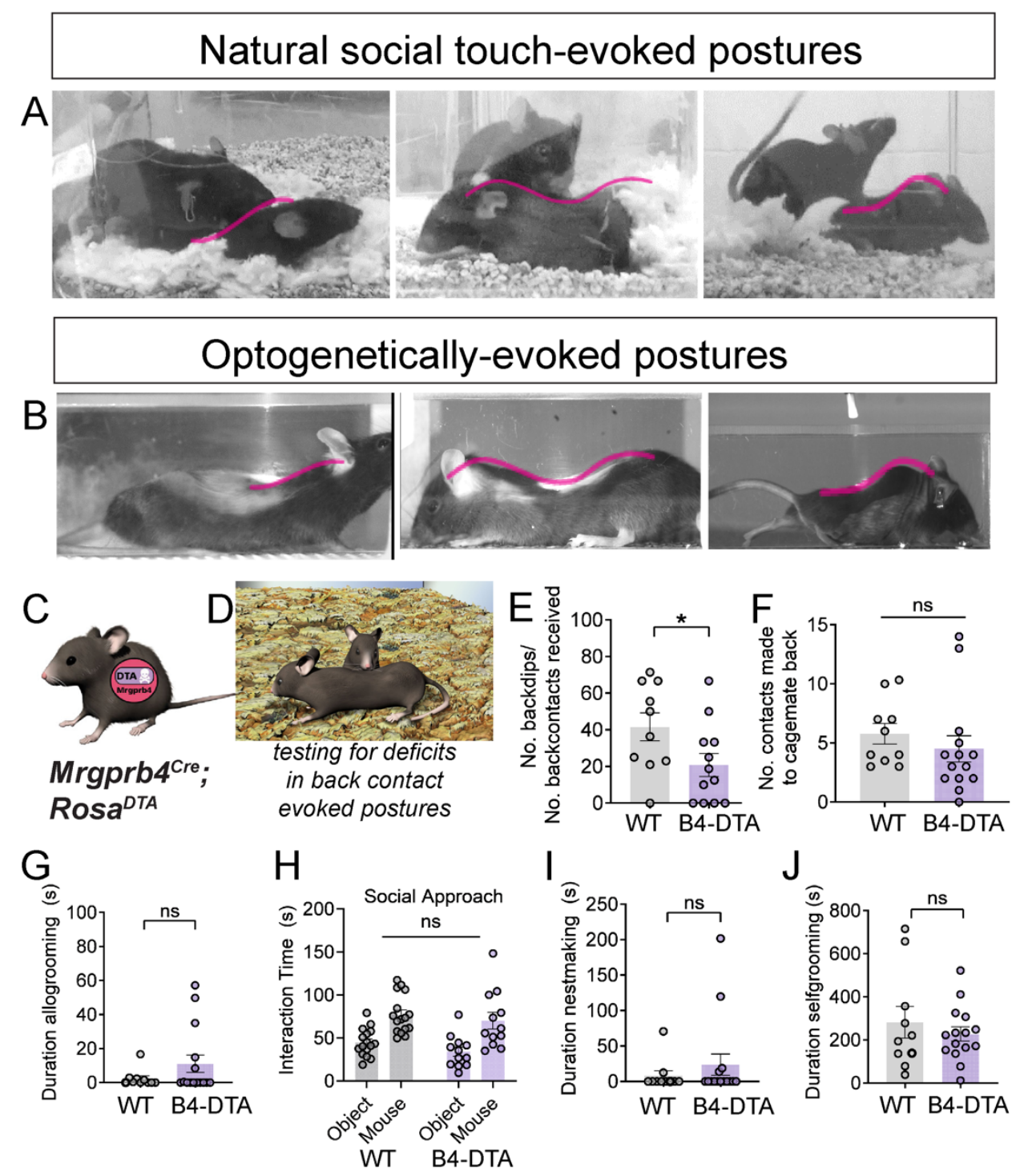

Figure 3: Mrgprb4-lineage neurons are required for female-to-female touch-evoked social postures but not general social interest. A) Still frames from behavioral videos showing the conspecific crawl posture: dorsiflexion in response to social touch. B) Still frames from highspeed videos showing the dorsiflexion induced by optogenetic activation of Mrgprb4-lineage neurons. These stills are eerily reminiscent of the postures induced by natural social touch. C) The Mrgprb4 ${ }^{C r e}$; Rosa ${ }^{D T A}$ females were used in this experiment. D) Graphic depicting a typical conspecific crawl backdip posture E) Number of conspecific crawl backdip occurrences per mouse divided by the total number of backcontacts that mouse received. Mrgprb4 ${ }^{\text {Cre }}$; Rosa ${ }^{D T A}$ females do fewer backdips than WT females $\left({ }^{*} P<0.05\right.$, unpaired t-test). F) Mrgprb4 ${ }^{\text {Cre }}$; Rosa ${ }^{\text {DTA }}$ females contact their cagemates' backs just as frequently as WT females contact their cagemates' backs in the 20 minute trial. G) Mrgprb4 $4^{\text {Cre }}$; Rosa ${ }^{\text {DTA }}$ females allogroom their cagemates for similar duration as controls. H) Mrgprb4 ${ }^{\text {Cre; }}$ Rosa ${ }^{D T A}$ females demonstrate similar preference for a mouse over an inanimate object compared to controls. I) Mrgprb4 $4^{C r e}$; Rosa ${ }^{D T A}$ females spend a similar duration nestmaking compared to controls. J) Mrgprb4Cre; Rosa ${ }^{D T A}$ females spend a similar duration self-grooming compared to controls.

conspecifc crawl was a dorsiflexion response to any social touch to the back: allogrooming, other taps or contacts to the back, or sliding underneath a stationary cage mate (example graphic Fig 3d). Self-grooming, initiating social touch, allogrooming, and nestmaking behaviors were similar between groups (Fig 3f-j). Relatedly, Mrgprb4Cre; 
Rosa ${ }^{D T A}$ mice display normal social interest as tested in a social approach assay when presented with the choice of interacting with another mouse or an inanimate object (Fig $3 \mathrm{~h}$ ). Thus, Mrgprb4-lineage neurons are not necessary for typical social development but are necessary for evoking touch-dependent social postures.

Chemogenetic activation of Mrgprb4-lineage neurons is not sufficient to promote social interaction or postures.

Next, we sought to determine whether activation of the Mrgprb4-lineage neurons could increase the frequency of conspecific crawl postures in a natural social setting. We therefore used a Designer Receptors Exclusively Activated by Designer Drugs (DREADD)-based chemogenetic approach to activate Mrgprb4-lineage neurons while avoiding experimenter intervention during the social paradigm, which would be required for transdermal optogenetic activation. We generated Mrgprb4Cre; Rosa ${ }^{\text {hM3dq }}{ }^{\text {mice }}$ and either Mrgprb4 ${ }^{C r e}$ or Rosa ${ }^{h M 3 d q}$ littermate controls and administered Clozapine N-oxide (CNO) or saline 30 minutes prior to reuniting the cage mates for the social interaction assay, as performed in Fig 3 (Supplemental Figure 4a-d). We observed that chemogenetic activation of Mrgprb4-lineage neurons did not promote increased social contact, an unsurprising result given that DTA-mediated ablation of Mrgprb4-lineage neurons did not impair social motivation or social contact (Supplemental Figure $4 \mathrm{f}, \mathrm{g}$ ). This further highlighted that Mrgprb4-lineage neurons, although recruited in touch-dependent social behaviors, do not appear to play a role in social motivation among same-sex conspecifics. Interestingly, however, there was no increase in social conspecific crawl postures (Supplemental Figure 4e). While optogenetic activation of Mrgprb4-lineage neurons is sufficient to induce a dorsiflexion posture in isolation, chemogenetic activation was not sufficient to promote the posture in social settings. It may be that focal activation on the back is required to generate the dorsiflexion, and body-wide chemogenetic activation is not a physiologically relevant sensory input for this social posture.

\section{Optogenetic-induced dorsiflexion posture is not modulated by ovarian hormones}

Decades of work have established that rodent lordosis is tightly modulated by levels of estrogen and progesterone ${ }^{25,31,32}$. To determine whether the dorsiflexion caused by optogenetic activation of Mrgprb4-lineage neurons posture represents a posture of sexual receptivity, we tested whether the posture was modulated by ovarian hormones. First, we tracked the extent of dorsiflexion with the natural estrous cycle determined by daily vaginal lavage. We found that the dorsiflexion posture during $20 \mathrm{~s}$ of optogenetic stimulation did not significantly vary across estrous states (Supplemental Figure 5a). Secondly, we determined whether experimental manipulation of ovarian hormones would impact the posture. We measured the optogenetic-induced postures in either vehicle or hormone-replaced ovariectomized females (to mimic a natural state of behavioral estrus). Consistent with the results for the natural estrous cycle, we found that the extent of the posture was not significantly different in ovariectomized compared to OVX + hormone replaced females (Supplemental Figure 5b). Thus, the dorsiflexion that results from the optogenetic activation of Mrgprb4-lineage neurons does not represent a state of sexual receptivity. Instead, the optogenetic activation of Mrgprb4-lineage neurons in the back 
skin may be triggering a local sensorimotor circuit that is recruited and modulated by hormonal conditions during natural sexual encounters.

Optogenetic activation of Mrgprb4-lineage neurons is sufficient to cause dopamine release

We hypothesized that Mrgprb4-lineage neurons may directly transduce rewarding sensation during sexual encounters. Because tactile input from the male to the female during sexual behavior occurs to the back and anogenital region, we examined the skin in both regions for Mrgprb4+ nerve endings. Consistent with prior neuroanatomical studies $^{1}$, Mrgprb4+ terminals innervated all examined hairy skin, including the back, underbelly, and skin surrounding the genitalia, but were absent from the glabrous lining of the vaginal wall (Figure 1r; Supplemental Figure 6). We next coupled fiber photometry with transdermal optogenetic stimulation to test whether activation of Mrgprb4-lineage neurons - in either the back or anogenital skin - is sufficient to induce dopamine release in nucleus accumbens (NAc), a brain region for dopaminergic sexual reward ${ }^{33-36}$ (Figure $4 a)$. To measure dopamine release we used stereotactic viral injections of the GRABDA dopamine sensor into the NAc. The GRAB ${ }_{\mathrm{DA}}$ sensor is a GPCR-based approach where dopamine release and binding to its cognate receptor generates fluorescence with subcellular resolution and sub-second kinetics that we can detect with a photodetector ${ }^{37}$. Mrgprb4 ${ }^{\text {Cre }}$; Rosa ${ }^{C h R 2 / C h R 2}$ females expressing the GRAB ${ }_{D A}$ dopamine sensor in NAc were given either 30s transdermal optogenetic stimulation to the back or anogenital region or the same treatment with non-stimulating green light while dopamine signals were recorded. When we analyzed the $\mathrm{GRAB}_{\mathrm{DA}}$ signal upon optogenetic back stimulation of Mrgprb4-lineage neurons, we did not observe a significant increase in dopamine release (Supplemental Figure 7). We next turned to the Mrgprb4-lineage neurons innervating the skin surrounding the genitalia, which are expected to be stimulated during male intromission into the vagina during mounting. Intriguingly, blue light stimulation to skin surrounding the genitalia, but not green, was sufficient to raise GRABDA deltaF/F significantly from baseline levels (Fig 4b-e). This demonstrates that the Mrgprb4-lineage neurons in the anogenital skin are sufficient, in otherwise socially-isolated mice, to activate reward circuitry.

\section{Mrgprb4-lineage neurons are required for dopamine release during sexual behavior}

The Mrgprb4-lineage neurons are both necessary for the positive reinforcement of female sexual receptivity and sufficient to cause dopamine release in isolated females. We hypothesized that Mrgprb4-lineage neurons may be driving dopamine release during sexual behaviors. To determine whether Mrgprb4-lineage neurons are indeed required for sexual reward, we injected Mrgprb4 ${ }^{\text {Cre }}$; Rosa ${ }^{D T A}$ females and littermate controls with the GRAB $B_{D A}$ dopamine sensor to observe any differences in dopamine activity during sexual behavior in the absence of Mrgprb4-lineage neurons (Figure 4f). We measured dopamine activity in the seconds surrounding mounts, anogenital sniffs, and other instances of back contacts to the female. We found that Mrgprb4 ${ }^{C r e} ; R_{\text {Rosa }}{ }^{D T A}$ females had significantly reduced dopamine signal immediately following mount initiation compared to 

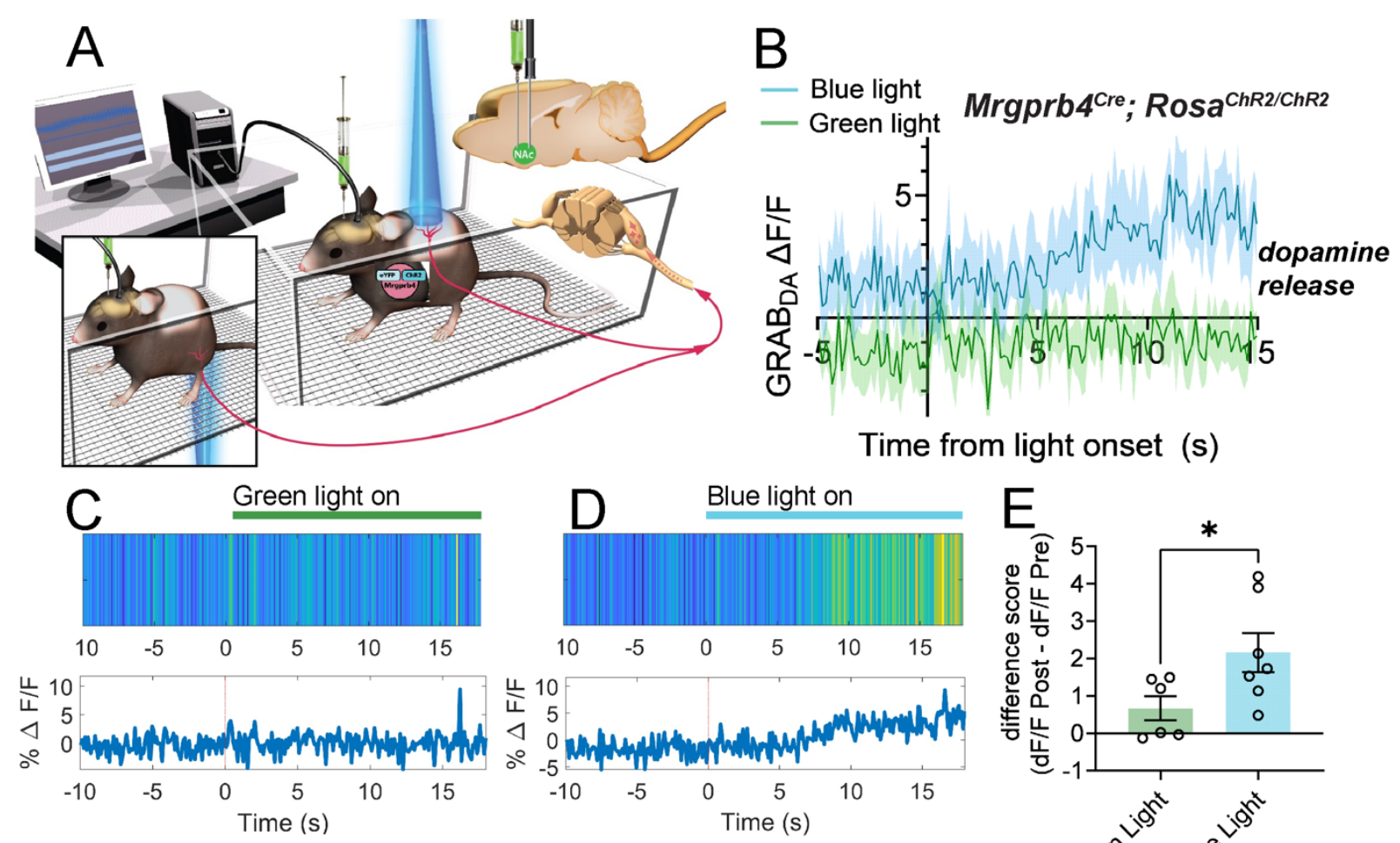

Mrgprb4 ${ }^{\mathrm{Cre}}$; Rosa ${ }^{\mathrm{ChR2} / \mathrm{ChR2}}$
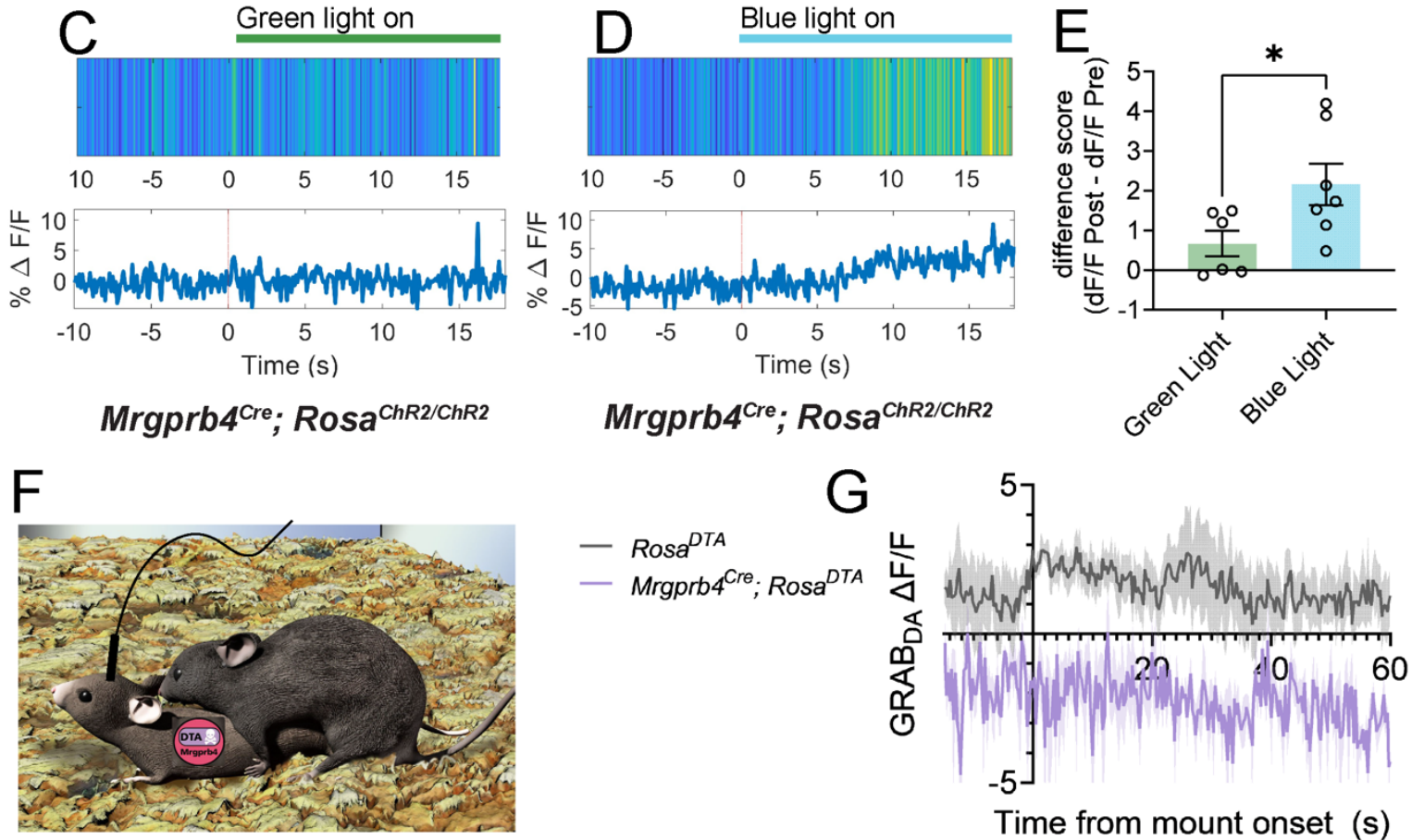

Mrgprb4 ${ }^{\text {Cre }}$ Rosa ${ }^{\text {ChR2/ChR2 }}$
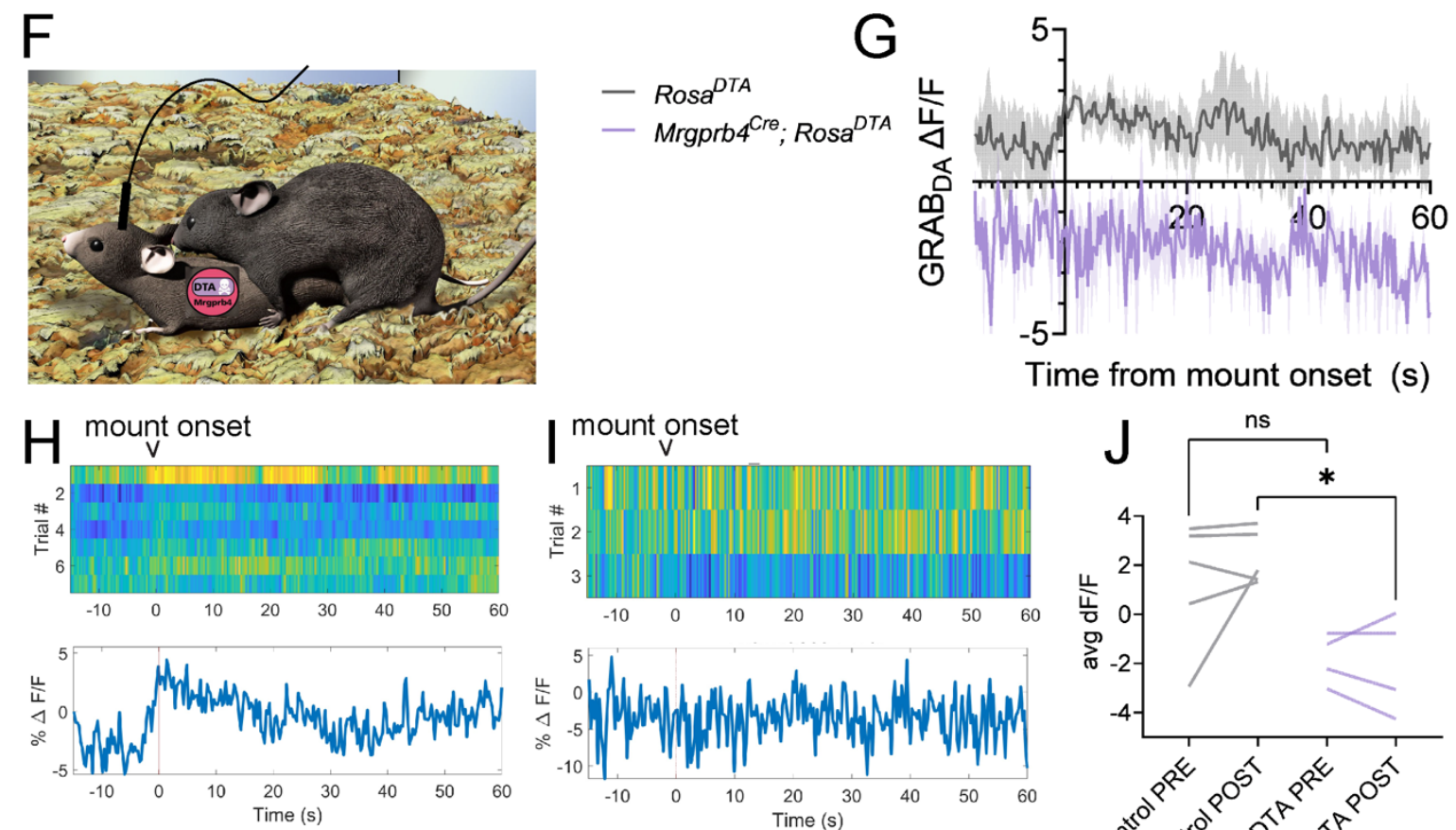

Rosa $a^{\text {DTA }}$

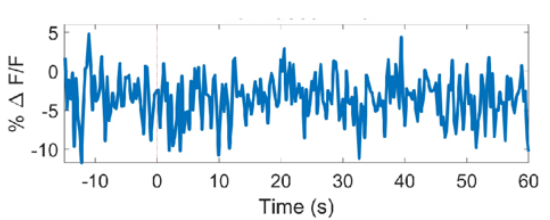

Mrgprb4 $^{\text {Cre }} ;$ Rosa $^{\text {DTA }}$
Time from mount onset (s)

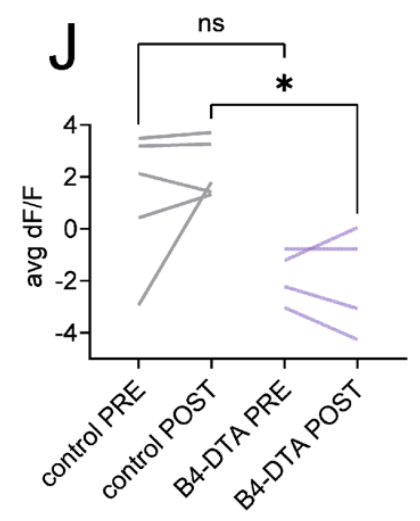

littermate controls, suggesting the Mrgprb4-lineage neurons are indeed required for the sexual reward that occurs during a male mount (Fig 4g-j). No significant difference in dopamine activity was detected for anogenital sniffs or back contacts (Supplemental Figure 8). Mrgprb4-lineage neurons may signal reward in these social touch scenarios, 
Figure 4: Mrgprb4-lineage neurons trigger dopamine release and are required for dopamine release during sexual mounts. A) Schematic depicting the experimental setup. Female Mrgprb4 ${ }^{\mathrm{Cre}}$; Rosa ${ }^{\mathrm{ChR} 2 / \mathrm{ChR} 2}$ mice with shaved backs, which had been injected with $\mathrm{GRAB}_{\mathrm{DA}}$ to the NAc two weeks prior to testing, were placed under plastic chambers on a mesh platform. Pulsed blue (stimulating) or green (control) laser light ( $35 \mathrm{~mW}, 10 \mathrm{~Hz}$ sin wave) was shined to either the back skin or skin surrounding the vagina while recording GRAB DA $_{\text {signals. B) Average GRAB }}$ DA delta F/F signals $(\mathrm{N}=6-7)$ for green and blue light. $\mathrm{C})$ Representative trace from a mouse with green light shined to the skin surrounding the vagina, light stimulation begins $T=0$. D) Representative trace from the same mouse with blue light shined to the skin surrounding the vagina. E) Average deltaF/F pre stim (-5-0) subtracted from average deltaF/F post stim (10-15s) is significantly greater for blue light stimulation compared to the green light control, $\left({ }^{*} P<0.05\right.$, unpaired $\mathrm{t}$-test). $\left.\mathrm{F}\right)$ Graphic depicting experimental setup. Female Mrgprb4 ${ }^{C r e}$; Rosa ${ }^{D T A}$ mice or littermate Rosa ${ }^{D T A}$ controls, which had been injected with GRAB $B_{D A}$ to the NAc over two weeks prior to testing were paired with males for a mating assay. Females were ovariectomized and hormone primed to be in a state of behavioral estrus for testing. GRAB $B_{\mathrm{DA}}$ signals were recorded for the entire pairing and analyzed surrounding three events: mount, anogenital sniff to female, and back contact to female. G) Average deltaF/F traces surrounding mount onset ( $\mathrm{T}=0)$ for Mrgprb4 $^{\text {Cre }}$; Rosa ${ }^{D T A}$ females (purple) or littermate Rosa ${ }^{D T A}$ controls (gray) $(\mathrm{N}=4-5)$. H) Representative average GRAB ${ }_{D A}$ signal across seven mounts in one pairing for a Rosa ${ }^{D T A}$ control, mount onset at $\mathrm{T}=0$. I) Representative average $\mathrm{GRAB}_{\mathrm{DA}}$ signal across three mounts in one pairing for a Mrgprb4 ${ }^{\text {Cre }}$; Rosa ${ }^{D T A}$ female. J) The average GRAB $\mathrm{BA}_{\mathrm{DA}}$ signal following mount onset is reduced in Mrgprb4 ${ }^{\text {Cre }}$; Rosa ${ }^{D T A}$ females compared to Rosa ${ }^{D T A}$ controls ( ${ }^{*} P<0.05$, One way ANOVA).

but dopamine release may be at levels too low to detect a difference, compared to the robust dopamine release in females upon male mounts. Together, these data reveal that Mrgprb4-lineage neurons communicate with the brain's reward center during socially rewarding sexual behavior.

\section{Discussion}

Although affective social touch begins at the skin's surface, the molecular identity of sensory neurons in the skin that detect socially relevant signals and pass them to the central nervous system has remained unknown. Moreover, because touch itself is highly heterogeneous (i.e., discriminative touch to detect texture with our fingertips versus the affiliative touch during a hug from a friend), sensory perception is likely generated by different sets of neurons to provide specificity $8,12,38-43$. Armed with this information, where does one begin the search for touch neurons underlying social reward, including sexual receptivity? Within the deep ocean of DRG neuron types, one class, termed C-low threshold mechanoreceptors (C-LTMRs), or C-tactile afferents in humans, are implicated in detecting gentle strokes across the skin's surface ${ }^{7}$. Although a limited number of papers in the mouse identify molecular populations of C-LTMRs, including their neuroanatomy and roles in somatosensation during baseline and chronic pain states ${ }^{10,12,44-49}$, the role of C-LTMRs in promoting social behaviors remains obscure.

Here, we demonstrate that Mrgprb4-lineage neurons are indeed critical for specific social behaviors and for signaling social reward to the brain. Focal activation of Mrgprb4lineage neurons yields a striking dorsiflexion posture resembling mammalian lordosis, representing the first acute behavioral response to optogenetic activation of social touch neurons. Mrgprb4-lineage neurons are required for two touch-dependent social postures: sexual behavior and crawling under same-sex conspecifics. However, their role in female sexual behavior is not simply in the local lordotic reflex; rather, the neurons convey an 
affective sensation that reinforces sexual receptivity, and without them, male advances during sexually receptive hormonal states become aversive. This finding suggests that Mrgprb4-lineage neurons contribute to the perceived valence of sexual encounters in females by encoding the rewarding aspect of male sexual touch.

Lastly, we use fiber photometry to functionally link Mrgprb4-lineage neurons in the skin to reward circuitry in the brain. Transdermal optogenetic activation of Mrgprb4lineage neurons in the skin surrounding female genitalia is sufficient to induce dopamine release in the NAc, representing the first demonstration of molecularly defined somatosensory neurons triggering activation of a brain reward center. Moreover, Mrgprb4-lineage neurons are required for this same dopamine release during male mounts. These experiments have revealed the sufficiency of peripheral inputs to regulate social reward independent of context and other sensory cues. Since ventral tegmental dopaminergic neurons that project to the NAc are themselves functionally heterogeneous ${ }^{50,51}$, it is possible that some of these neurons might be tuned for encoding rewarding touch. It will also be interesting to determine if Mrgprb4-lineage neurons are involved in other behaviors that integrate social touch, such as maternal care. Moreover, the approaches outlined here could be leveraged to determine the functional roles of other molecularly defined touch neurons. We believe this study not only draws new attention to the importance of elucidating skin-to-brain circuits, but also unveils the therapeutic potential of peripheral manipulations for enhancing intact or impaired social reward systems, including sexual receptivity, or simulating social reward during periods of isolation. 


\section{Supplemental Figure 1}
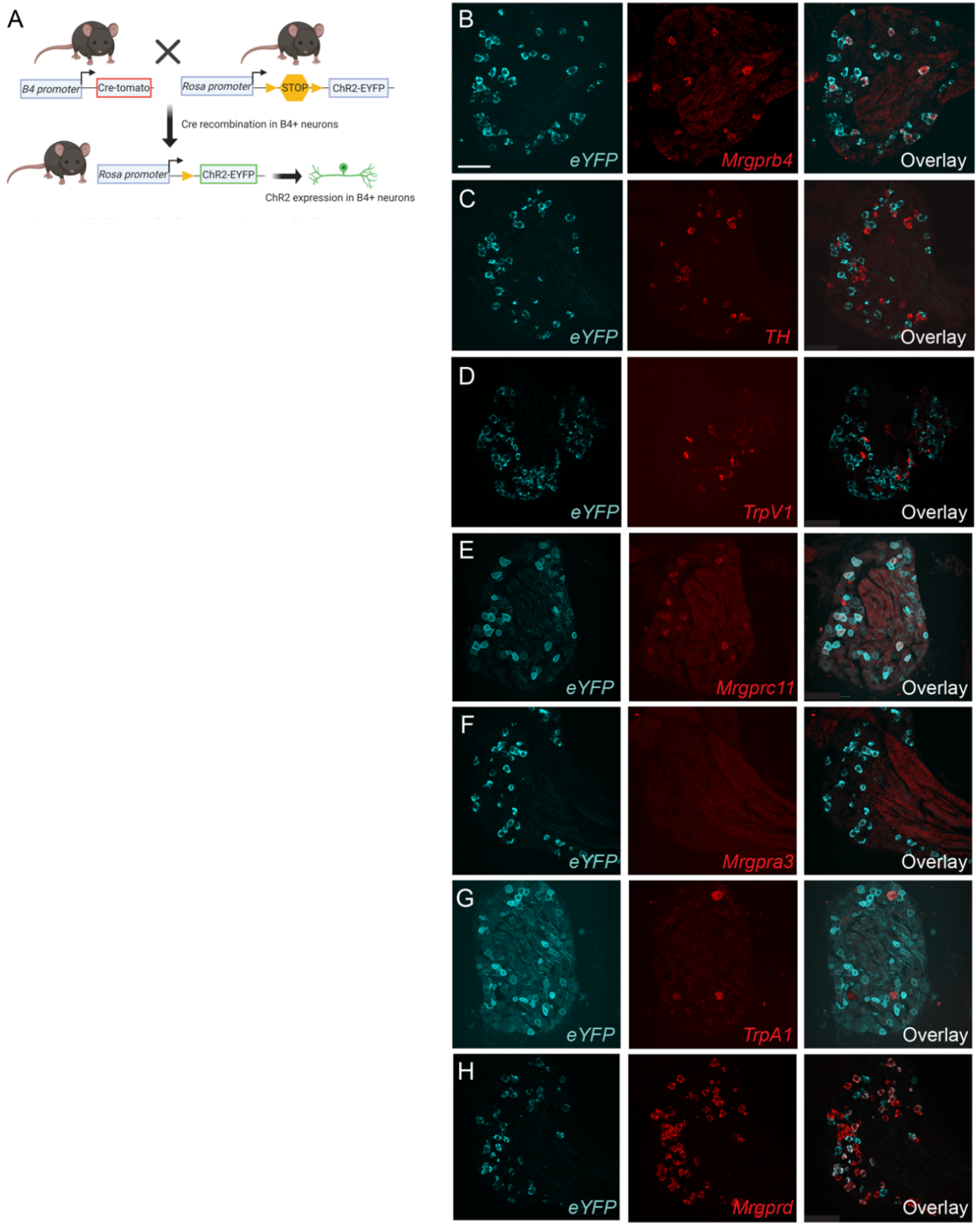

Supplemental Figure 1. Characterizing expression of ChR2-eYFP in Mrgprb4-lineage neurons. A) Schematic showing breeding strategy to express ChR2-eYFP in Mrgprb4-lineage neurons. B-H) Double RNA in situ hybridization with RNA Scope probe that detect eYFP (cyan) or another marker gene (red). Quantification and description of results in Figure 1d,e. Scale bar represents $100 \mu \mathrm{m}$. 


\section{Supplemental Figure 2}

A

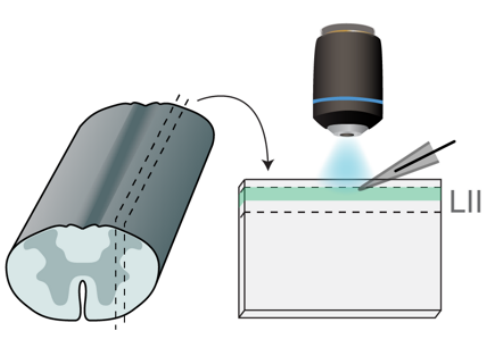

B

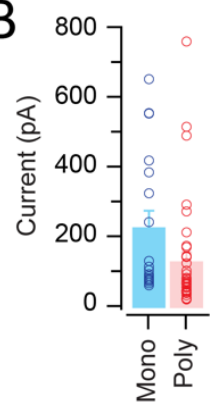

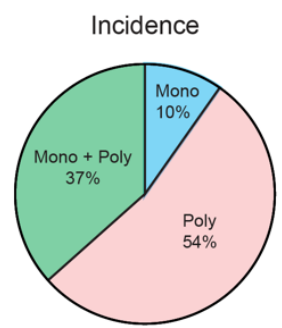

.
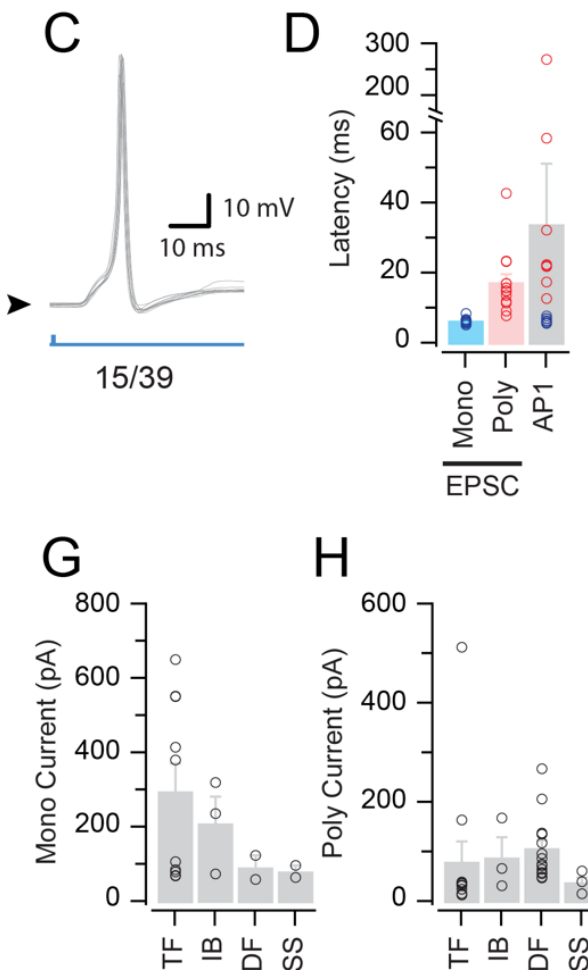

$\mathrm{H}$

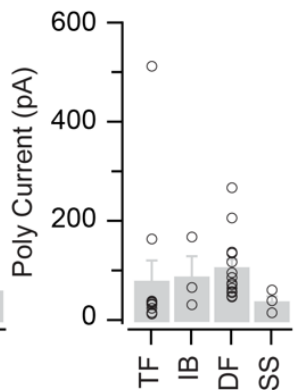

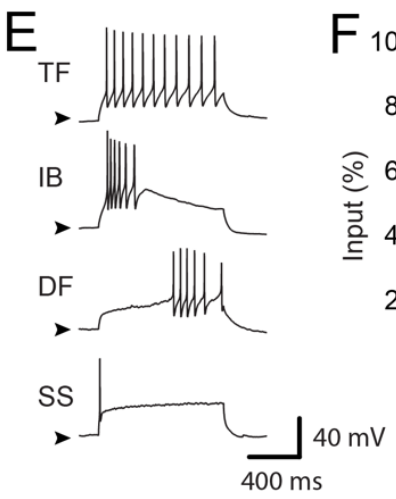

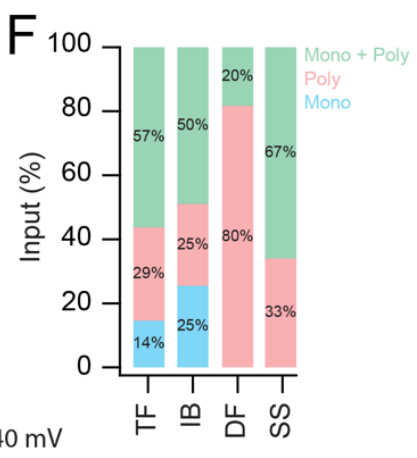

1

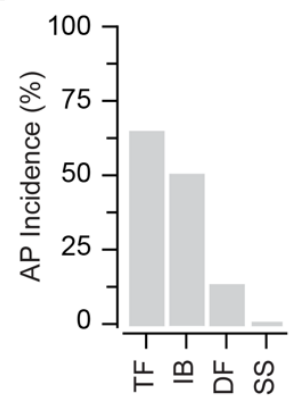

Supplemental Figure 2. Characterizing physiological properties of dorsal horn spinal cord neurons that receive synaptic input from Mrgprb4-lineage neurons. A) Schematic of the spinal cord slice preparation to activate Mrgprb4+ terminals with blue light in the superficial dorsal horn and record from synaptically connected neurons. B) Left shows grouped data of mono and polysynaptic EPSC amplitudes. Right shows incidence of a monosynaptic only, polysynaptic only, or mono + polysynaptic input. C) Optically evoked action potential (AP). 15/39 neurons tested fired AP's following $1 \mathrm{~ms}$ photostimulation. D) Latency of oEPSCs (mono and polysynaptic) in these neurons, compared to the latency of the first evoked AP. Of these 15 neurons, 7 display characteristics of AP's evoked by direct Mrgprb4 afferent input, 8 display characteristics of AP's evoked by indirect (polysynaptic) Mrgprb4 inputs. E,F) In a subset of recordings AP discharge was characterized in post-synaptic neurons $(n=36)$. 2/14 Tonic firing (TF) neurons received mono input only, $4 / 14$ poly only, and $8 / 14$ both. 0/15 Delayed firing (DF) neurons received mono input only, $12 / 15$ received poly only, and $3 / 15$ both. $1 / 4$ Initial bursting (IB) neurons received mono input only, $1 / 4$ received poly only, and 2/4 both. 0/3 Single spikers (SS) received mono input only, $1 / 3$ received poly only, and $2 / 3$ both. G) TF neurons receive the strongest monosynaptic inputs, followed by IB's. H) Polysynaptic currents are similar across all types. I) AP incidence is highest in TF neurons. 


\section{Supplemental Figure 3}

\section{Mrgpra3 $^{\text {Cre; }}$ Rosa ${ }^{\text {ChR2/ChR2 }}$}

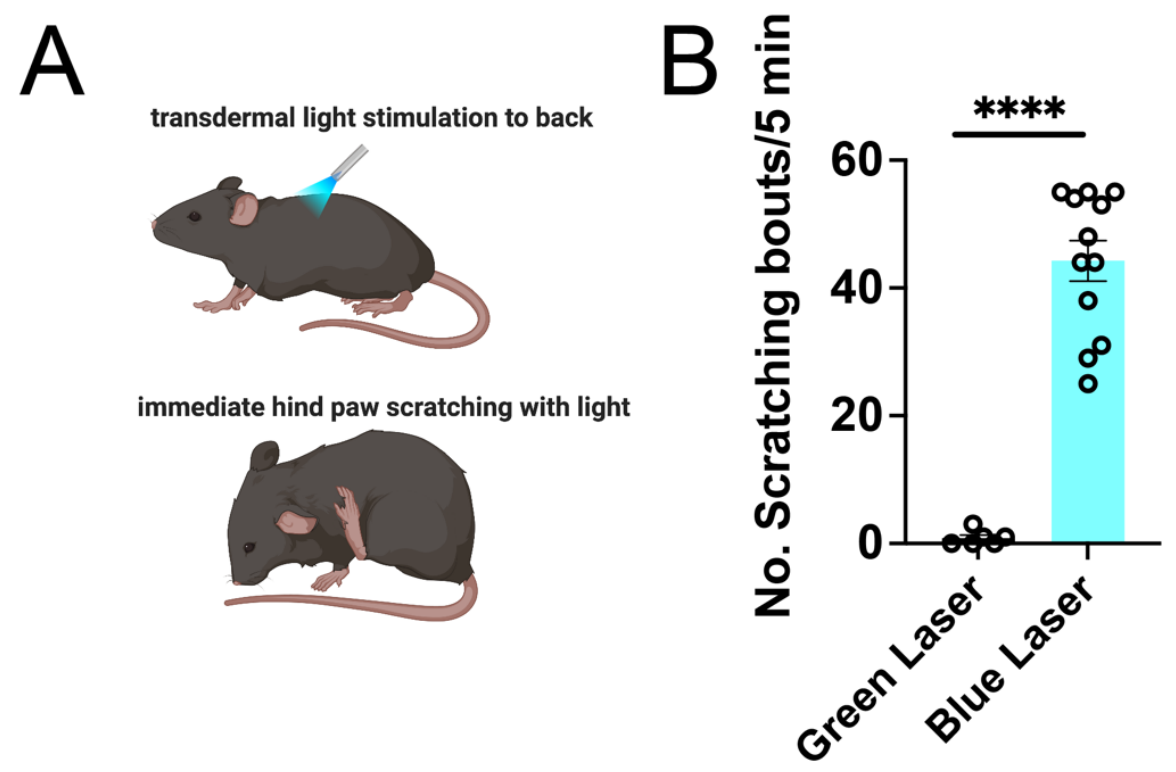

Supplemental Figure 3. Transdermal optogenetic activation of Mrgpra3+ neurons elicits stereotyped scratching behaviors. A) Schematic showing experimental setup with blue light applied to the shaved back skin, which elicits nearly immediate scratching bouts, and not the back lowering dorsiflexion phenotype observed when the same experiments are performed with Mrgprb4-lineage neurons. B) Quantification of scratching bouts to 5 minutes of blue or green laser light (negative control). Unpaired student's t-test, ${ }^{* * * *} P \leq 0.0001$. Individual circles represent a single mouse. 


\section{Supplemental Figure 4}
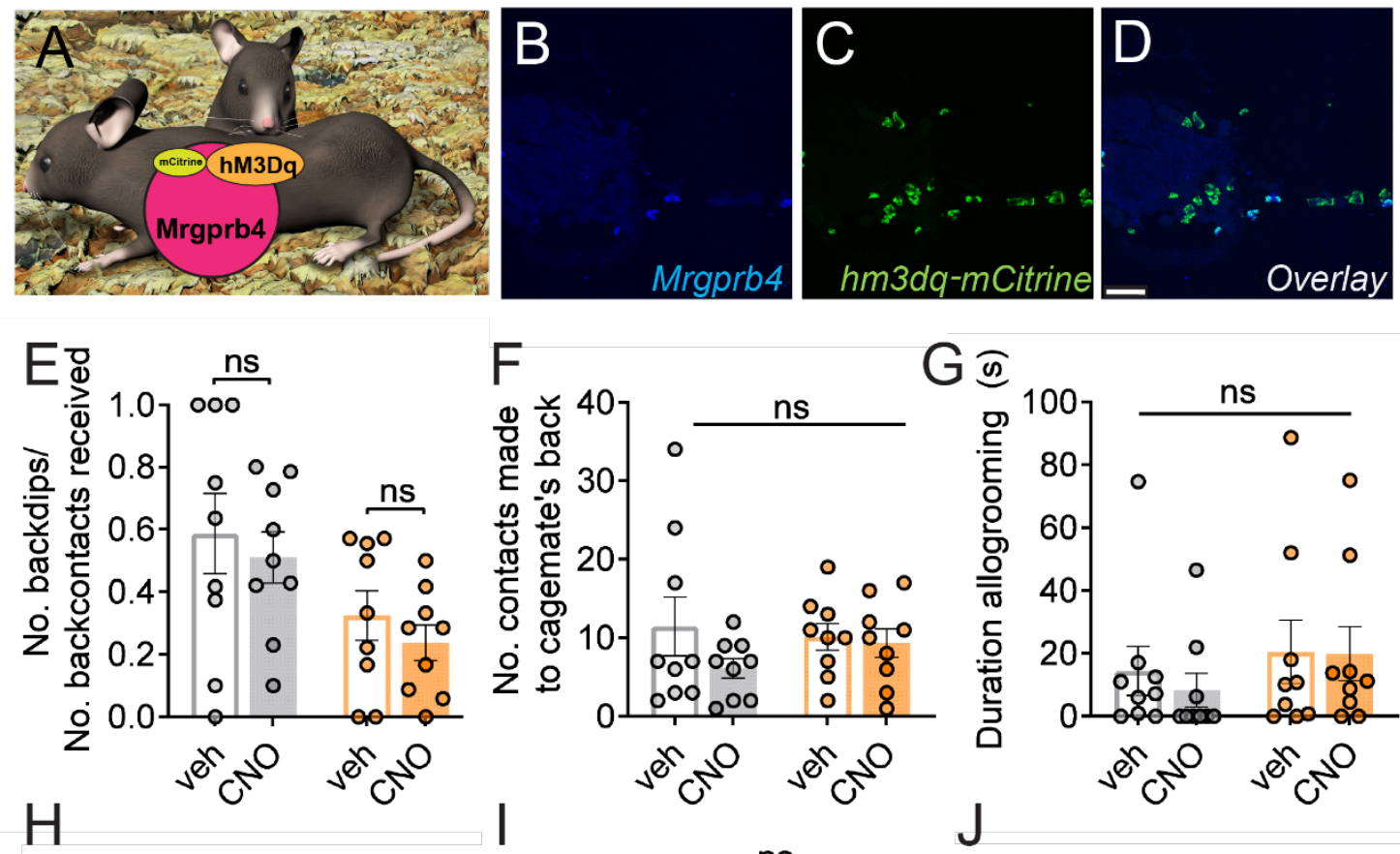

Gळ
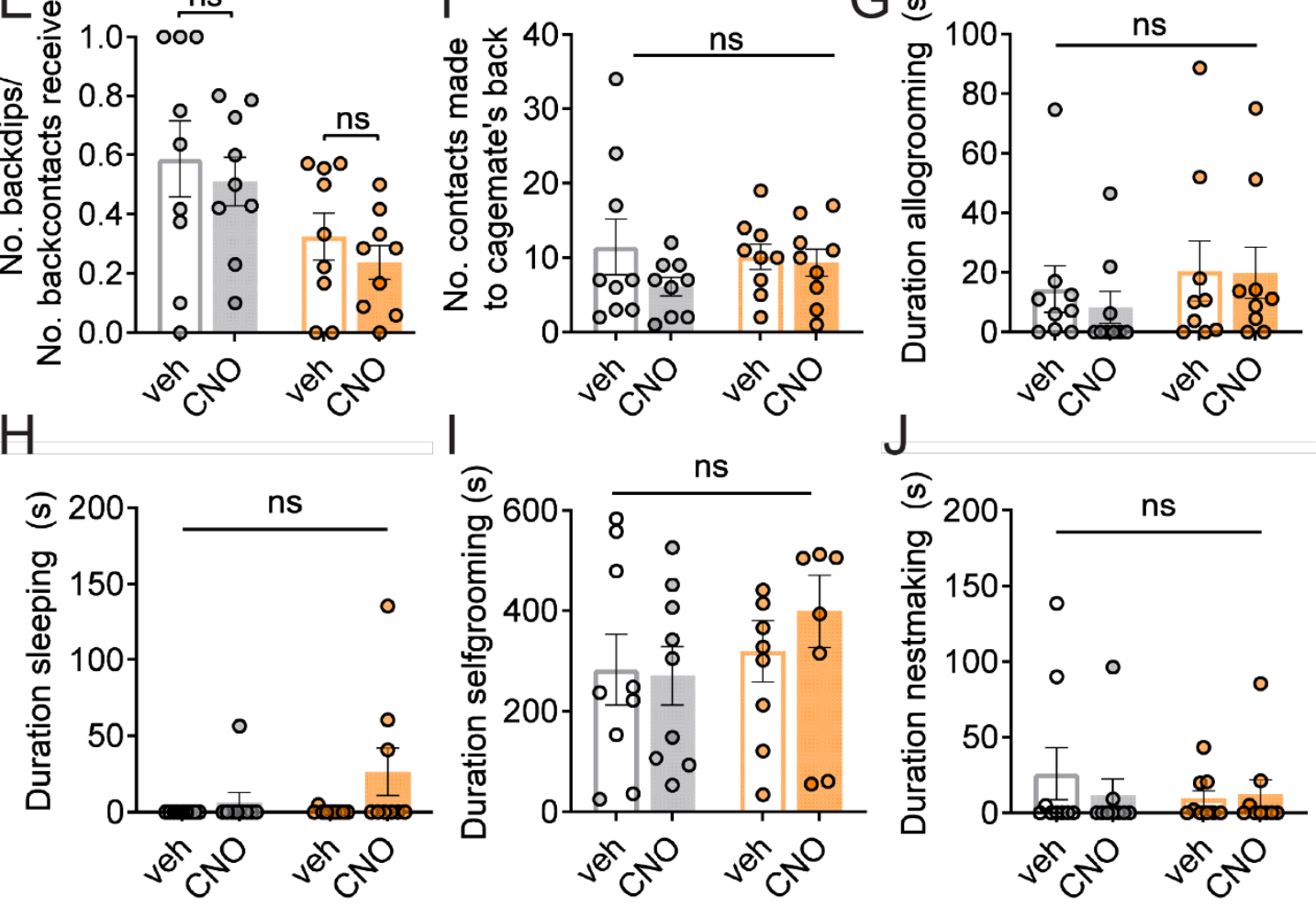

Supplemental Figure 4. Body-wide chemogenetic activation of Mrgprb4-lineage neurons does not alter social behaviors. A) Schematic showing chemogenetic strategy to activate Mrgprb4-lineage neurons with DREADDmediated activation while assessing social behaviors. B-D) Confirmation of targeting strategy to insert the DREADD receptor hM3Dq-mCitrine into Mrgprb4-lineage neurons. Data visualized with RNA Scope in situ hybridization. Scale bar represents $75 \mu \mathrm{m}$. E-J) No statistical difference in any assay measuring social (E-G) or other (H-J) behaviors. All data plotted as mean +/- SEM, with one-way ANOVA followed by Tukey's post-hoc comparisons. 


\section{Supplemental Figure 5}
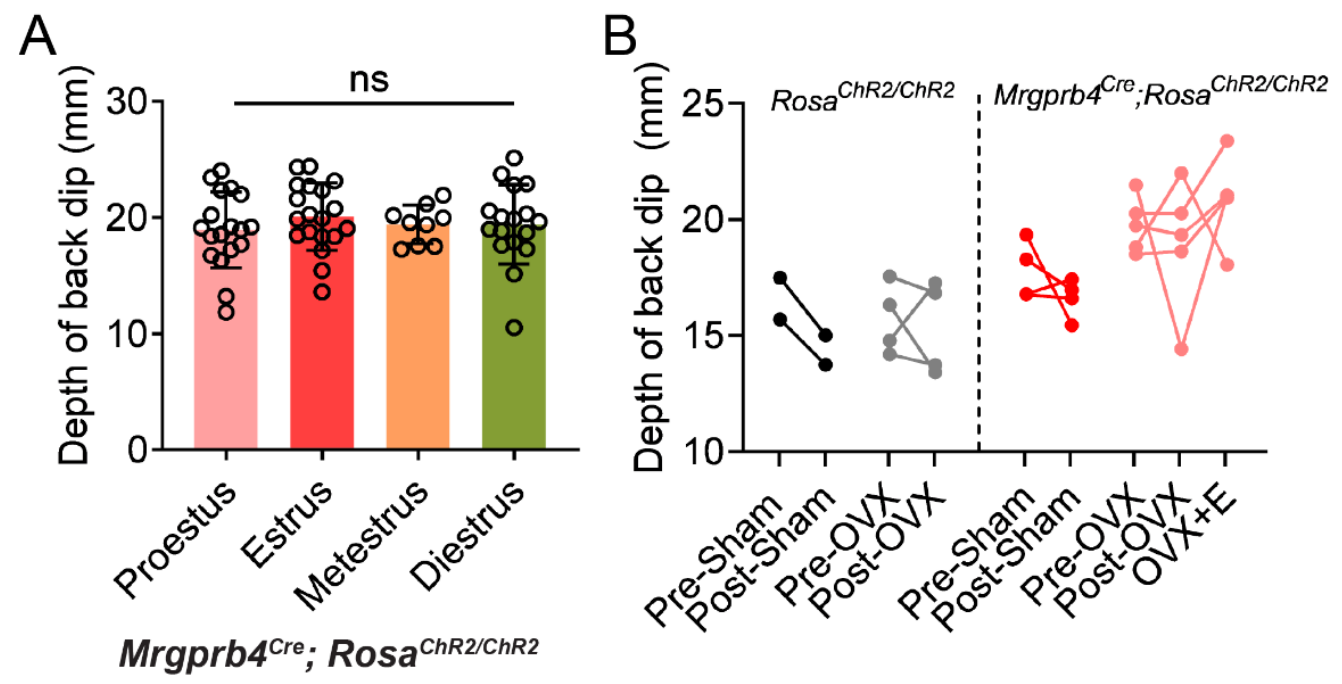

\footnotetext{
Supplemental Figure 5. Lordosis-like dorsiflexion posture observed in Mrgprb4 ${ }^{C r e} ; R^{\circ o s a}{ }^{C h R 2 / C h R 2}$ female mice is independent of estrous state. A) No differences in the depth of the optogenetically induced back dip across stages of the estrous cycle. Circles represent individual mice, data plotted as mean +/- SEM. B) Neither ovariectomizing (OVX) females, nor replacing estrogen and progesterone exogenously to mimic behavioral estrus, significantly modulates the optogenetically induced back dip. Dots represent individual mice. All data analyzed by one way ANOVA followed by Tukey's post-hoc comparisons.
} 


\section{Supplemental Figure 6}

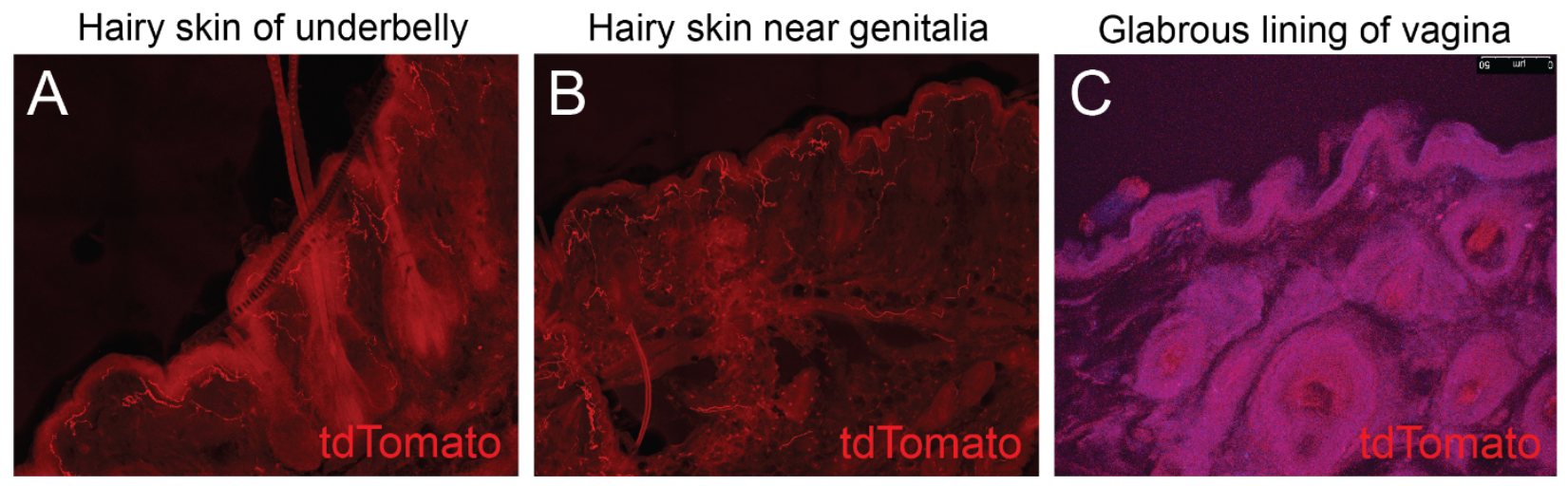

\section{Mrgprb4 ${ }^{\text {Cre-tdTomato; Rosa }}{ }^{C h R 2 / C h R 2}$}

Supplemental Figure 6. Nerve terminal endings of Mrgprb4+ neurons in skin of the anogenital regions with immunostaining of tdTomato. $A, B$ ) Nerve terminal endings in red are seen in the underbelly skin as well as the hairy skin surrounding the female genitalia. C) Nerve terminals for Mrgprb4+ touch neurons are not seen in the vaginal wall, which is glabrous, non-hairy skin. 


\section{Supplemental Figure 7}

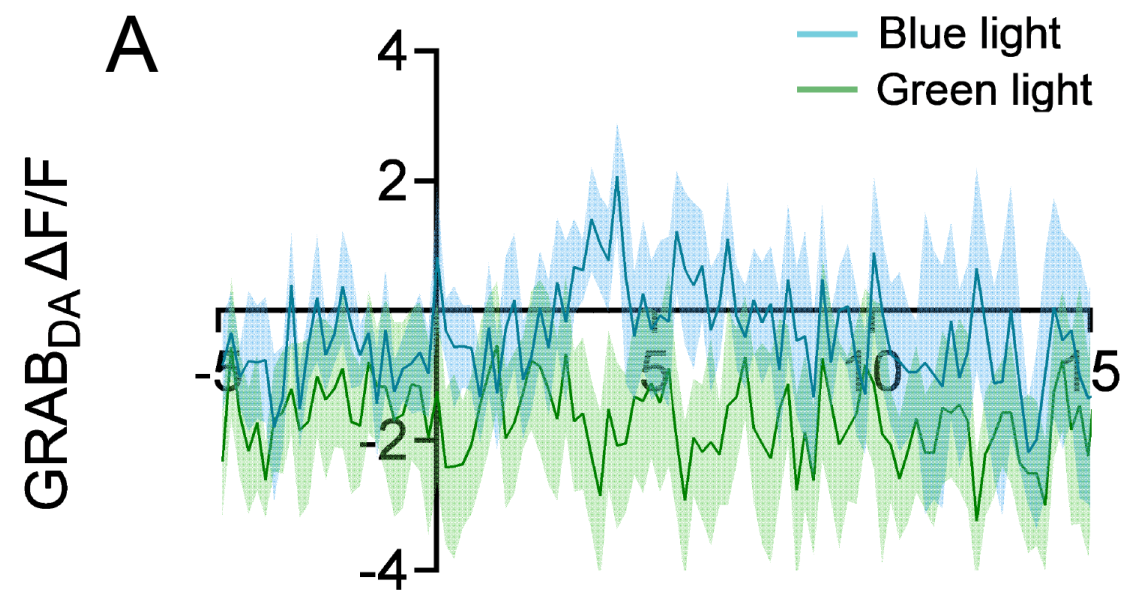

Time from light onset (s)
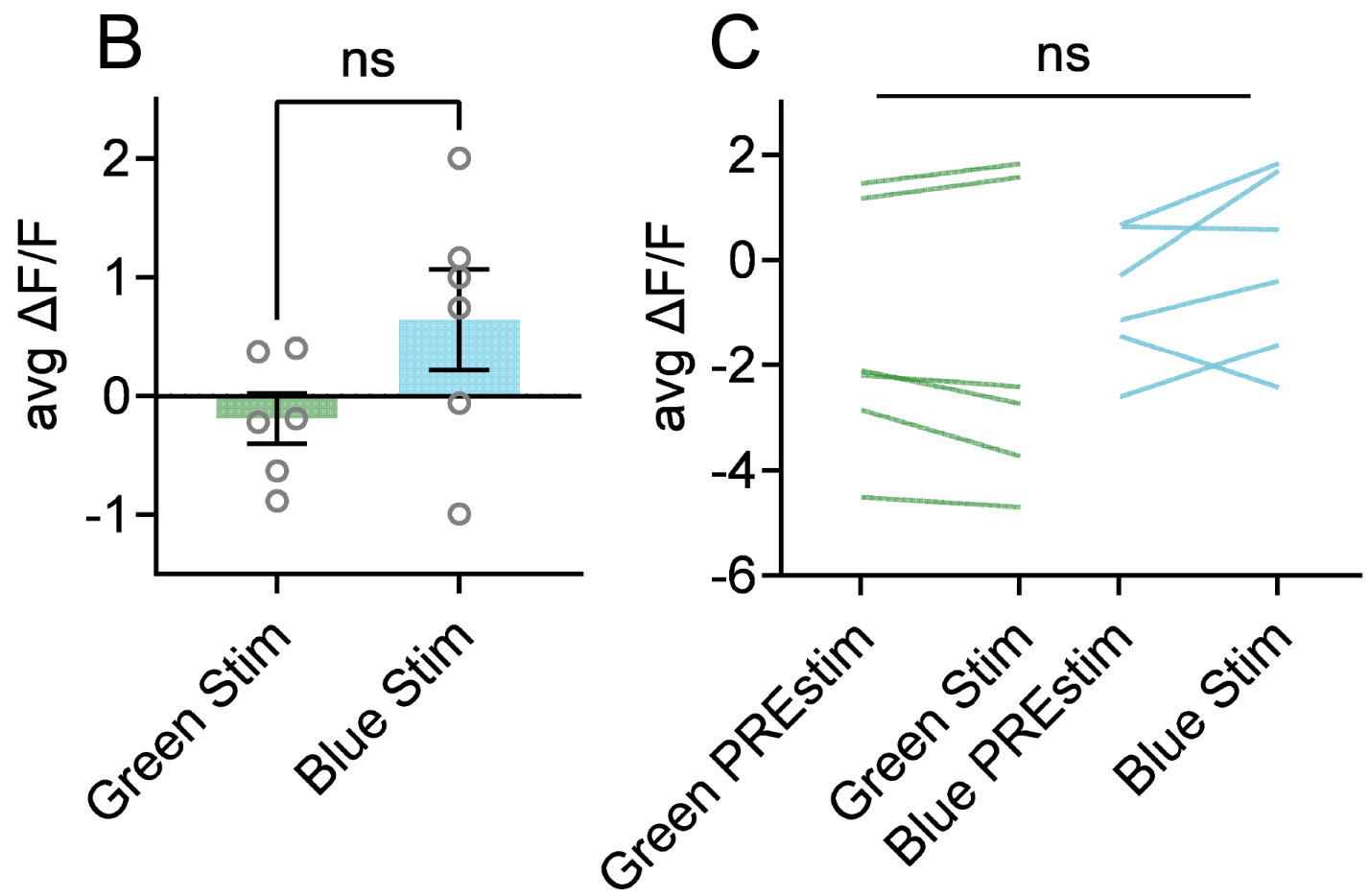

Supplemental Figure 7. Optogenetic activation of Mrgprb4-lineage neurons in the back skin does not lead to detectable dopamine release. A) Average $\mathrm{GRAB}_{\mathrm{DA}}$ delta $\mathrm{F} / \mathrm{F}$ signals $(\mathrm{N}=6-7)$ for green and blue light. $\left.\mathrm{B}\right)$ Average deltaF/F pre stim (-5-0) subtracted from average deltaF/F post stim (10-15s) is not statistically significantly different for blue light stimulation compared to the green light control, unpaired t-test. C) Average deltaF/F pre and post stim are not statistically different between blue and green light control stimulation, one-way ANOVA. 


\section{Supplemental Figure 8}

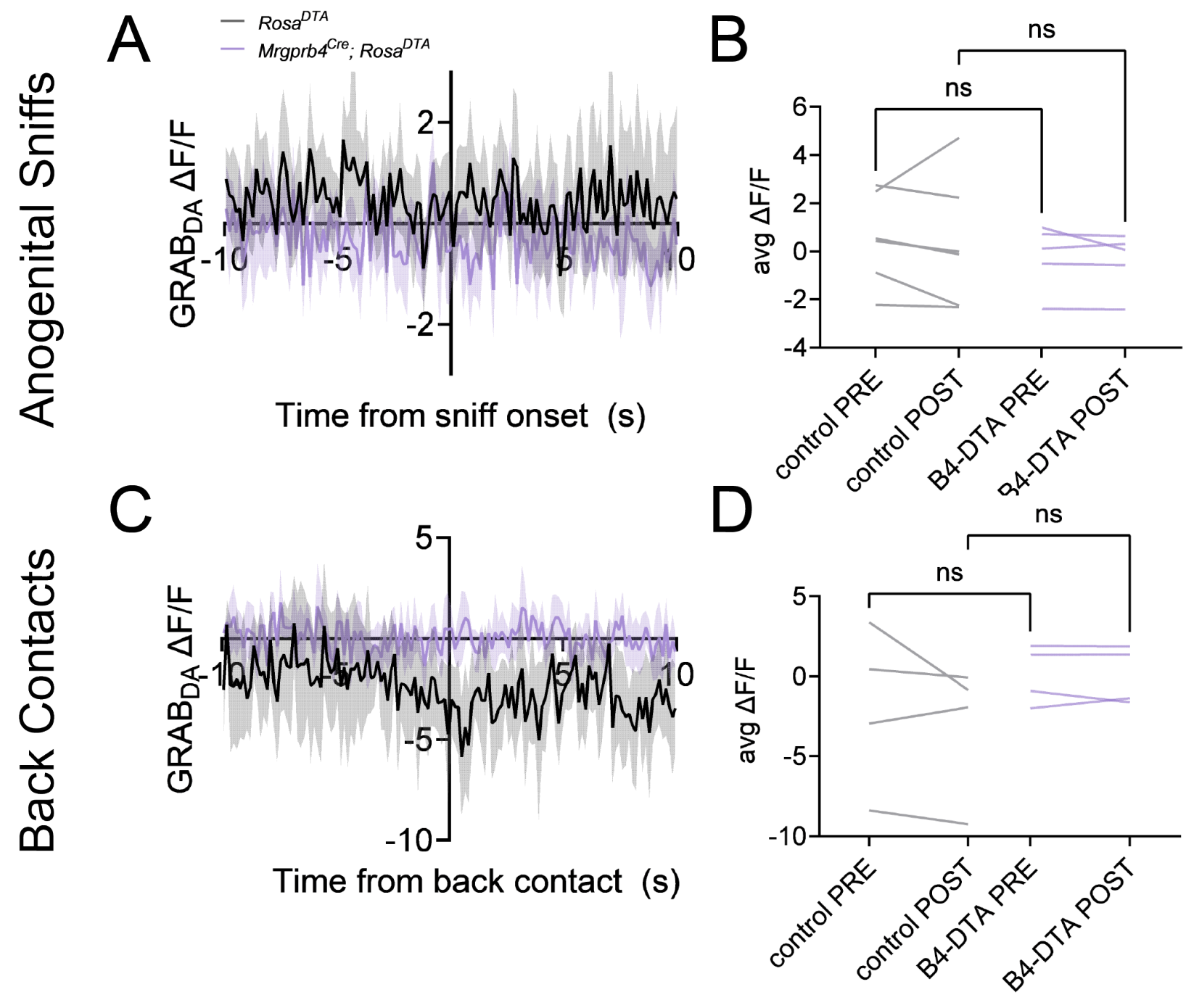

Supplemental Figure 8. No changes in dopamine release in females observed in control or Mrgprb4-neuron ablated mice to anogenital sniffs or back contacts. A,C) Average deltaF/F traces $(N=4-6)$ in the 20s surrounding $(A)$ anogenital sniffs or $(C)$ back contacts received from the male during the sexual encounter. B,D) Average deltaF/F pre (-5-0s) and post (0-10s) anogenital sniff $(B)$ or back contact $(D)$ are not statistically different between B4-DTA and control female mice, one-way ANOVA. 


\section{Methods}

Mice and Behavioral Testing

All testing was performed in compliance with the NIH Guide for the Care and Use of Laboratory Animals and were approved by the Institutional Animal Care and Use Committee of the University of Pennsylvania and Columbia University. Mice born into our colony on a C57bl/6J background were maintained in conventional housing with food and water available ad libitum when not being tested, on a 12 hour light cycle beginning at 7:00, and all testing unless otherwise noted occurred during the light cycle in a room directly adjacent to the housing room in the animal facility. Mouse lines used in this study are located at Jackson Laboratories: C57BL/6J (Stock No: 000664), Mrgprb4 ${ }^{\text {Cre }}$ (Stock No: 021077), Mrgprd ${ }^{C r e-E R T 2}$ (Stock No: 031286), RosaChR2-eYFP (Stock No: 024109), $\operatorname{Rosa}^{D T A}$ (Stock No: 009669), Rosa ${ }^{\text {Ga-DREADD }}$ (Stock No: 026220). The MrgprA3 ${ }^{\text {Cre }}$ line was generously provided by Dr. Xinzhong Dong at Johns Hopkins University School of Medicine.

RNA Scope in situ hybridization

Brains were harvested immediately following transcardial perfusion and post-fixed in $4 \%$ PFA in PBS at $4^{\circ} \mathrm{C}$ for $24 \mathrm{~h}$. Brains were subsequently submerged in $30 \%$ sucrose in PBS at $4^{\circ} \mathrm{C}$ for $18 \mathrm{~h}$ or until sunk, flash frozen in cryomold on dry ice in OCT, sectioned at $12 \mu \mathrm{m}$ directly onto Superfrost Plus Slides and stored in foil-wrapped slide box at $-80^{\circ} \mathrm{C}$ until beginning Fixed Frozen RNA Scope protocol (ACD).

Entire spinal columns were harvested immediately following transcardial perfusion and post-fixed in $4 \%$ PFA in PBS at $4^{\circ} \mathrm{C}$ for $24 \mathrm{~h}$. Either spinal cord or DRG were subsequently dissected and submerged in $30 \%$ sucrose overnight or until sunk. Tissue was flash frozen in cryomold on dry ice in OCT, sectioned at $12 \mu \mathrm{m}$ directly onto Superfrost Plus Slides, and stored in foil-wrapped slide box at $-80^{\circ} \mathrm{C}$ until beginning Fresh Frozen RNA Scope protocol (ACD). Note the fixation step was skipped in ACD's Fresh Frozen protocol for these tissues.

\section{Immunohistochemistry}

Brains, spinal cord, and DRG were collected and prepared in the same way as in situ hybridization. 30 $\mu \mathrm{m}$ cryosections were cut directly onto Superfrost Plus Slides. Slides were frozen overnight in a slide box at $-80^{\circ} \mathrm{C}$. Slides were washed $3 \times 10 \mathrm{~min}$ in PBS; 30 minutes in PBST; 1 hour in PBST with $5 \%$ normal donkey serum. Primary antibody, 1:1000 in PBST with 5\% normal donkey serum, was applied to slides in a humidified chamber overnight at room temperature. Secondary antibody, 1:400 in PBST with 5\% normal donkey serum, was applied to slides in a humidified chamber 1-2 hours at room temperature. Slides were washed 3x 10 minutes in PBS before applying mounting media and coverslip.

Transdermal optogenetic activation of Mrgprb4-lineage neurons 
Transdermal optogenetic stimulation of sensory neurons was performed as previously described (Abdus-Saboor et al., 2019). In brief, 8-16 week old female mice were habituated to mesh platform under a plastic chamber for 1 hour on each of two days prior to behavioral testing. Experimenter was present with lights, camera, and laser running during habituation to mimic entire sensory experience of test day. On the day of testing, the mice were habituated to the chambers for an additional 20 minutes before stimulation. $35 \mathrm{~mW}$ blue laser light pulsed at $10 \mathrm{~Hz}$ sin wave was shined through the ceiling of the chamber to the shaved backs of mice for 20 seconds during high-speed video captured at $750 \mathrm{fps}$.

\section{Quantification of the back dip}

Optogenetic dorsiflexion posture was calculated as the maximum back dip from the ceiling of the behavioral chamber for the duration of the 20 s stimulation. High speed videos were analyzed in Image $J$ to track the lowest point of the back throughout the $20 \mathrm{~s}$ stimulation. The lowest $y$ value was subtracted from the $y$ value of the top of the chamber, and the value was converted from pixels to millimeters by determining the pixel height of the $4.5 \mathrm{~cm}$ chamber for each video. It was decided that this was the most quantifiable way to characterize the dip. While duration or frequency of response require a subjective determination of when a back dip starts and stops - and therefore can be more challenging to compare to control animals - the back dip depth is the most objective measure because it allows us to compare between natural movement of the spine in controls, small back dips, and most drastic back dips. This measure encapsulates the full variability of response.

\section{Conditioned Place Preference}

8-14 week old Mrgprb4 ${ }^{\text {Cre; }}$ Rosa ${ }^{\text {ChR2/ChR2 females or Mrgprd }}{ }^{\text {CRE-ERT2; RosaChR2/ChR2 and }}$ Cre-negative RosaChR2/ChR2 female littermates underwent a 9 day conditioned place preference paradigm. The apparatus consisted of two chambers, one paired with almond extract and a textured floor, the other coconut extract and a smooth floor. Additionally, one chamber wall had stripes, while the other had polka dots. These olfactory and visual stimuli were present throughout the 9 day paradigm to aid in the mouse's encoding of different chambers. Days 1-3 were habituation: each mouse was allowed 20 minutes to explore the two chambered apparatus with no optogenetic stimulation. Days 4-8 were training: each mouse was allowed 20 minutes to freely move about the two-chambered apparatus, now receiving laser light stimulation to the back. In one chamber the mouse received $10 \mathrm{~Hz}$ pulsed sin wave $35 \mathrm{~mW}$ blue laser light to the shaved back, and the other chamber non-stimulating green light of the same parameters. Experimenter held the laser lights $\sim 1 \mathrm{~cm}$ from the back skin for the duration that the mouse was in the chamber. The lasers were held with an extendable alligator clip to avoid casting body shadow on the chambers. Day 9 was test day: each mouse was allowed 20 minutes to freely move about the two-chambered apparatus in the absence of any laser light stimulation. Baseline preference was calculated for each mouse by averaging the duration spent in each chamber across the three habituation days. The conditioned preference was calculated 
for each mouse as the duration spent in each chamber on the test day. Percent change was calculated as percent time in blue light chamber after training - percent time in blue light chamber before training.

\section{Determination of natural estrous state}

Natural estrous cycle was determined by vaginal lavage. Immediately following behavioral testing, the vagina was flushed with $20 \mu \mathrm{l}$ ultrapure water which was then pipetted onto a Superfrost plus slide for examination under dissecting scope. Mice were tested midmorning each day to ensure the most accurate tracking. Lavage samples were assessed as described ${ }^{52}$, and estrous state was recorded throughout the week to ensure normal cycling. Data from mice with lavage samples that could not be fit into a typical four or five day cycling pattern were excluded.

\section{Ovariectomy Surgery}

Ovariectomies were performed on 8 week old female mice under $1.5-2 \%$ isoflurane using proper sterile technique. $5 \mathrm{mg} / \mathrm{kg}$ oral or intraperitoneal meloxicam and $2 \mathrm{mg} / \mathrm{kg}$ subcutaneous bupivacaine (at incision sites) were administered before surgery. A $0.5 \mathrm{~cm}$ incision is made $1 \mathrm{~cm}$ lateral to spinal cord, at the point where ribcage ends. An equivalent incision was made through the muscle wall. The white fat pad was exposed to identify the ovary, which was cauterized with a hemostat and scraped off with a scalpel. The fat pad was reinserted and 1-2 sutures closed the muscle wall. 2-4 sutures closed the skin. Meloxicam was administered 24 hours after surgery and mice were allowed two weeks to recover before behavioral testing.

\section{Lordosis Quotient Assay}

10-14 week old ovariectomized females underwent two overnight pairings with stud males two weeks following surgery. To mimic behavioral estrus state at the time of pairing, females were subcutaneously injected with $0.5 \mu \mathrm{g}$ estradiol benzoate in sunflower seed oil both 52 and 28 hours before pairing, and with $800 \mu$ progesterone in sesame oil 4 hours before pairing. The females received the same hormone treatment prior to the lordosis quotient assay. Lordosis quotient assay was conducted in male home cage, 1-2 hours into the dark cycle. Video of the assay was recorded for 20 minutes or 20 attempted male mounts, whichever came first. Lordosis quotient was calculated as the number of female receptive responses divided by the number of attempted male mounts. A receptive response for the female was scored as all four limbs securely on the cage floor with no combative or escape behaviors. If the first male did not mount within 10 minutes, the female was moved to another male's cage. For any given trial, up to three males may have been used. Receptive postures were scored for quality on a scale from 1-3 as follows: 1: limbs on the ground with no attempts to escape, neither dorsiflexion nor upturned nose. 2: Some dorsiflexion, no upturned nose. 3: Robust dorsiflexion and/or upturned nose. Posture scores were averaged within each trial.

Interfemale Social Behavior Assay 
The interfemale social behavior assay was adapted from allogrooming assays $s^{53,54}$ and conducted in the same way for both diphtheria toxin-mediated ablation and chemogenetic activation of Mrgprb4-lineage neurons. 8-14 week old cage mate females (from different litters but shared cage for at least three weeks prior to testing) were acclimated to the behavioral room and brief experimenter handling for 1 hour the day prior to testing. Immediately prior to testing, the mice were separated in clean cages for 30 minutes to promote social interaction upon reunion. For chemogenetic experiments, the mice were injected with either saline or $0.5 \mathrm{mg} / \mathrm{kg} \mathrm{CNO}$ in saline immediately prior to the 30 minute separation so that behaviors would be scored 40-60 minutes after injection, when CNO has peak effects. The two females were reunited in the home cage for 30 minutes, during which video was recorded. The first 10 minutes served as habituation, during which time the mice predominantly explored the cage, and the following 20 minutes were scored for behaviors. Experimenter, blinded to genotype/treatment group, scored videos for duration allogrooming, duration selfgrooming, duration nestmaking, duration sleeping, number of conspecific crawl behaviors, and number of contacts received to the back. BORIS software was used for this behavioral scoring.

\section{Viral injections and optic fiber implantation}

7-10 week old females were pretreated with $5 \mathrm{mg} / \mathrm{kg}$ oral or intraperitoneal meloxicam before surgery. They were anesthetized in a chamber with $3 \%$ isoflurane before being placed in a stereotaxic frame and kept anesthetized with $1.5-2 \%$ isoflurane. Skull was exposed and leveled before drilling at the appropriate coordinates. 200nL pAAV-hSynGRAB_DA1h (Addgene) was injected unilaterally into NAc (AP: $+1.0 \mathrm{~mm}$ relative to Bregma, ML: $\pm 1.2 \mathrm{~mm}$ relative to Bregma, DV: $4.6 \mathrm{~mm}$ from the brain surface) using a backfilled glass needle and syringe pump (PHD Ultra, Harvard Apparatus). Skin was either sutured for one week recovery before implanting optic fiber (Doric, MFC_200/2300.57_6mm_MF1.25_FLT) $200 \mu \mathrm{m}$ above injection site, or optic fiber was inserted immediately following injection. Three skull screws were inserted in the skull to stabilize the implant. A small amount of Metabond cement (Parkell) was used to bond the fiber, skull, and skull screws. Dental cement was applied on top of dried Metabond to create a stable implant structure. Mice were given 1 week to recover from implant surgery and 2 weeks to recover from injection and implant combined surgeries before behavioral testing. Meloxicam was administered 24 hours after surgery during post-operative monitoring.

\section{Fiber Photometry}

Zirconia sleeves (Doric) were used to connect the optic fiber implant to the patch cord. Signals were recorded using a real-time processor (RZ10X, TDT) and extracted in real time using Synapse software (TDT). A 465nm LED was used to excite the GRAB DA1h $_{\text {h }}$ while a $405 \mathrm{~nm}$ LED was used to measure changes in fluorescence due to photobleaching and movement artifacts.

For fiber photometry during transdermal optogenetic activation experiments, mice were placed on the mesh platform in plastic chambers with a $0.7 \mathrm{~cm}$ slot cut into the ceiling to 
allow the cord to connect from the head to the computer. After 30 second baseline recording, $10 \mathrm{~Hz}$ pulsed $\sin$ wave at $35 \mathrm{~mW}$ blue light was shined through the mesh platform to the vaginal area at a $2-3 \mathrm{~cm}$ distance for 30 seconds.

For fiber photometry during sexual behavior, females were placed in male home cage 1 hour into the dark cycle for 10 minutes or 10 mounts, whichever occurred first. If the male did not mount in the first 5 minutes, the female was placed in another male cage, for up to three total males.

TDT folders were imported directly into Fiber photometry Modular Analysis Tool (pMAT) software for analysis ${ }^{55}$. DeltaF/F was plotted as a peri-event time histogram from -10s to $20 \mathrm{~s}$, where $0 \mathrm{~s}$ is the start of optogenetic stimulation to the anogenital region. deltaF/F calculated by normalizing to the median of 5 s baseline sampling window $(-10--5 s)$, bin constant: 150s.

\section{Electrophysiology}

Recordings were made from Mrgprb4 ${ }^{\mathrm{Cre}} ; \mathrm{Rosa}^{\mathrm{ChR} 2 /+}$ or Mrgprb4Cre;RosaChR2/ChR2 mice (female; age $3.9 \pm 0.6 \mathrm{wks}$ ). Mice were anaesthetized with ketamine (100 mg/kg i.p), decapitated, and spinal cord (T10-L2) rapidly removed in ice-cold sucrose substituted artificial cerebrospinal fluid (sACSF) containing (in mM): 250 sucrose, $25 \mathrm{NaHCO}_{3}, 10$ glucose, $2.5 \mathrm{KCl}, 1 \mathrm{NaH}_{2} \mathrm{PO}_{4}, 6 \mathrm{MgCl}_{2}$, and $1 \mathrm{CaCl}_{2}$. Sagittal slices $(200 \mu \mathrm{m}$ thick) were prepared using a vibrating microtome (Leica VT1200S). Slices were incubated for at least $1 \mathrm{hr}$ at $22-24^{\circ} \mathrm{C}$ in an interface chamber holding oxygenated ACSF containing (in $\mathrm{mM}$ ): $118 \mathrm{NaCl}, 25 \mathrm{NaHCO}_{3}, 10$ glucose, $2.5 \mathrm{KCl}, 1 \mathrm{NaH}_{2} \mathrm{PO}_{4}, 1 \mathrm{MgCl}_{2}$, and $2.5 \mathrm{CaCl}_{2}$.

Following incubation, slices were transferred to a recording chamber and continually superfused with ACSF bubbled with Carbogen $\left(95 \% \mathrm{O}_{2}\right.$ and $\left.5 \% \mathrm{CO}_{2}\right)$ to achieve a pH of 7.3-7.4. All recordings were made at room temperature $\left(22-24^{\circ} \mathrm{C}\right)$ and neurons visualized using a Zeiss Axiocam 506 color camera. Recordings were acquired in voltage-clamp (holding potential $-70 \mathrm{mV}$ ) or current-clamp $(-60 \mathrm{mV})$. Patch pipettes $(3-7 \mathrm{M} \Omega)$ were filled with a potassium gluconate-based internal solution containing (in $\mathrm{mM}$ ): $135 \mathrm{C}_{6} \mathrm{H}_{11} \mathrm{KO}_{7}, 8$ $\mathrm{NaCl}, 10 \mathrm{HEPES}, 2 \mathrm{Mg}_{2} \mathrm{ATP}, 0.3 \mathrm{Na}_{3} \mathrm{GTP}$, and $0.1 \mathrm{EGTA}, \mathrm{pH} 7.3$ (with $\mathrm{KOH}$ ). No liquid junction potential correction was made, although this value was calculated at $14.7 \mathrm{mV}$ (22 ${ }^{\circ} \mathrm{C}$ ). All data were amplified using a MultiClamp 700B amplifier, digitized online (sampled at $20 \mathrm{kHz}$, filtered at $5 \mathrm{kHz}$ ) using an Axon Digidata 1550B, and acquired using Clampex software. After obtaining the whole-cell recording configuration, series resistance, input resistance, and membrane capacitance were calculated (averaged response to $-5 \mathrm{mV}$ step, 20 trials, holding potential $-70 \mathrm{mV}$ ). Photostimulation intensity was suprathreshold (24 $\mathrm{mW}$ ), duration $1 \mathrm{~ms}$ (controlled by transistor-transistor logic pulses).

\section{Acknowledgments}

We thank members of the Abdus-Saboor and Abraira labs for helpful discussion and comments on this manuscript. We thank Oliver Hobert, Richard Axel, and Charles Zuker 
for helpful comments on this work and manuscript. We thank Nitsan Goldstein and members of the Betley lab for assistance with fiber photometry set up. We thank David Barker for suggestions with pMAT software. We thank members of LM's thesis committee, including Lori Flannagan-Cato and Gregory Corder for helpful suggestions on experiments. We thank Qin Liu for sharing Mrgprb4Cre; RosaChR2/ChR2 mice with us. We thank Janet Sinn-Hanlon for illustrations. LM and MS are supported by NIH NRSA grants from NINDS and NCCIH. IAS and lab members acknowledge support from startup funds provided by the University of Pennsylvania and Columbia University, National Institute of Health grant NIH/NIDCR R00-DE026807, and fellowships from the Rita Allen Foundation and Alfred P. Sloan Foundation. VEA and MG acknowledge support from startup funds from Rutgers University, Pew Charitable Trust, NIH R01 and K01 grants from NINDS, and Whitehall Foundation.

\section{References:}

1 Liu, Q. et al. Molecular genetic visualization of a rare subset of unmyelinated sensory neurons that may detect gentle touch. Nat Neurosci 10, 946-948, doi:10.1038/nn1937 (2007).

2 Vrontou, S., Wong, A. M., Rau, K. K., Koerber, H. R. \& Anderson, D. J. Genetic identification of $\mathrm{C}$ fibres that detect massage-like stroking of hairy skin in vivo. Nature 493, 669-673, doi:10.1038/nature11810 (2013).

3 Orefice, L. L. et al. Targeting Peripheral Somatosensory Neurons to Improve TactileRelated Phenotypes in ASD Models. Cell 178, 867-886 e824, doi:10.1016/j.cell.2019.07.024 (2019).

4 Orefice, L. L. et al. Peripheral Mechanosensory Neuron Dysfunction Underlies Tactile and Behavioral Deficits in Mouse Models of ASDs. Cell 166, 299-313, doi:10.1016/j.cell.2016.05.033 (2016).

5 Peled-Avron, L. \& Shamay-Tsoory, S. G. Don't touch me! autistic traits modulate early and late ERP components during visual perception of social touch. Autism Res 10, 1141-1154, doi:10.1002/aur.1762 (2017).

6 Pawling, R., Cannon, P. R., McGlone, F. P. \& Walker, S. C. C-tactile afferent stimulating touch carries a positive affective value. PLoS One 12, e0173457, doi:10.1371/journal.pone.0173457 (2017).

$7 \quad$ Ackerley, R. et al. Human C-tactile afferents are tuned to the temperature of a skinstroking caress. J Neurosci 34, 2879-2883, doi:10.1523/JNEUROSCI.2847-13.2014 (2014).

8 Handler, A. \& Ginty, D. D. The mechanosensory neurons of touch and their mechanisms of activation. Nat Rev Neurosci 22, 521-537, doi:10.1038/s41583-021-00489-x (2021).

9 Delfini, M. C. et al. TAFA4, a chemokine-like protein, modulates injury-induced mechanical and chemical pain hypersensitivity in mice. Cell Rep 5, 378-388, doi:10.1016/j.celrep.2013.09.013 (2013).

10 Lou, S., Duan, B., Vong, L., Lowell, B. B. \& Ma, Q. Runx1 controls terminal morphology and mechanosensitivity of VGLUT3-expressing C-mechanoreceptors. J Neurosci 33, 870-882, doi:10.1523/JNEUROSCI.3942-12.2013 (2013).

11 Liu, Y. et al. Mechanisms of compartmentalized expression of Mrg class G-proteincoupled sensory receptors. J Neurosci 28, 125-132, doi:10.1523/JNEUROSCI.447207.2008 (2008). 
$12 \mathrm{Li}, \mathrm{L}$. et al. The functional organization of cutaneous low-threshold mechanosensory neurons. Cell 147, 1615-1627, doi:10.1016/j.cell.2011.11.027 (2011).

13 Olson, W. et al. Sparse genetic tracing reveals regionally specific functional organization of mammalian nociceptors. Elife 6, doi:10.7554/eLife.29507 (2017).

14 Abdus-Saboor, I. et al. Development of a Mouse Pain Scale Using Sub-second Behavioral Mapping and Statistical Modeling. Cell Rep 28, 1623-1634 e1624, doi:10.1016/j.celrep.2019.07.017 (2019).

15 Beaudry, H., Daou, I., Ase, A. R., Ribeiro-da-Silva, A. \& Seguela, P. Distinct behavioral responses evoked by selective optogenetic stimulation of the major TRPV1+ and MrgD+ subsets of C-fibers. Pain 158, 2329-2339, doi:10.1097/j.pain.0000000000001016 (2017).

16 Warwick, C. et al. MrgprdCre lineage neurons mediate optogenetic allodynia through an emergent polysynaptic circuit. Pain 162, 2120-2131,

doi:10.1097/j.pain.0000000000002227 (2021).

17 Sun, S. et al. Leaky Gate Model: Intensity-Dependent Coding of Pain and Itch in the Spinal Cord. Neuron 93, 840-853 e845, doi:10.1016/j.neuron.2017.01.012 (2017).

18 Daou, l. et al. Optogenetic Silencing of Nav1.8-Positive Afferents Alleviates Inflammatory and Neuropathic Pain. eNeuro 3, doi:10.1523/ENEURO.0140-15.2016 (2016).

19 lyer, S. M. et al. Virally mediated optogenetic excitation and inhibition of pain in freely moving nontransgenic mice. Nat Biotechnol 32, 274-278, doi:10.1038/nbt.2834 (2014).

20 Daou, I. et al. Remote optogenetic activation and sensitization of pain pathways in freely moving mice. J Neurosci 33, 18631-18640, doi:10.1523/JNEUROSCI.2424-13.2013 (2013).

21 Sharif, B., Ase, A. R., Ribeiro-da-Silva, A. \& Seguela, P. Differential Coding of Itch and Pain by a Subpopulation of Primary Afferent Neurons. Neuron 106, 940-951 e944, doi:10.1016/j.neuron.2020.03.021 (2020).

22 Pfaff, D. W. \& Sakuma, Y. Facilitation of the lordosis reflex of female rats from the ventromedial nucleus of the hypothalamus. J Physiol 288, 189-202 (1979).

23 Pfaff, D. W. \& Sakuma, Y. Deficit in the lordosis reflex of female rats caused by lesions in the ventromedial nucleus of the hypothalamus. J Physiol 288, 203-210 (1979).

24 Kow, L. M., Montgomery, M. O. \& Pfaff, D. W. Triggering of lordosis reflex in female rats with somatosensory stimulation: quantitative determination of stimulus parameters. $J$ Neurophysiol 42, 195-202, doi:10.1152/jn.1979.42.1.195 (1979).

25 Larsson, K., Sodersten, P., Beyer, C., Morali, G. \& Perez-Palacios, G. Effects of estrone, estradiol and estriol combined with dihydrotestosterone on mounting and lordosis behavior in castrated male rats. Horm Behav 7, 379-390, doi:10.1016/0018506x(76)90009-x (1976).

26 Sodersten, P. \& Larsson, K. Lordosis behavior and mounting behavior in male rats: effects of castration and treatment with estradiol benzoate or testosterone propionate. Physiol Behav 14, 159-164, doi:10.1016/0031-9384(75)90160-2 (1975).

27 Tang, Y. et al. Social touch promotes interfemale communication via activation of parvocellular oxytocin neurons. Nat Neurosci 23, 1125-1137, doi:10.1038/s41593-0200674-y (2020).

28 McCarthy, E. A. et al. DREADD-induced silencing of the medial amygdala reduces the preference for male pheromones and the expression of lordosis in estrous female mice. Eur J Neurosci 46, 2035-2046, doi:10.1111/ejn.13636 (2017).

29 Frye, C. A., Walf, A. A., Kohtz, A. S. \& Zhu, Y. Progesterone-facilitated lordosis of estradiol-primed mice is attenuated by knocking down expression of membrane progestin receptors in the midbrain. Steroids $\mathbf{8 1}, 17-25$, doi:10.1016/j.steroids.2013.11.009 (2014). 
30 Brock, O., Baum, M. J. \& Bakker, J. The development of female sexual behavior requires prepubertal estradiol. J Neurosci 31, 5574-5578,

doi:10.1523/JNEUROSCI.0209-11.2011 (2011).

31 White, S. \& Uphouse, L. Estrogen and progesterone dose-dependently reduce disruptive effects of restraint on lordosis behavior. Horm Behav 45, 201-208, doi:10.1016/j.yhbeh.2003.10.001 (2004).

32 Yanase, M. \& Gorski, R. A. Sites of estrogen and progesterone facilitation of lordosis behavior in the spayed rat. Biol Reprod 15, 536-543, doi:10.1095/biolreprod15.4.536 (1976).

33 Beny-Shefer, Y. et al. Nucleus Accumbens Dopamine Signaling Regulates Sexual Preference for Females in Male Mice. Cell Rep 21, 3079-3088, doi:10.1016/j.celrep.2017.11.062 (2017).

34 Pfaus, J. G., Damsma, G., Wenkstern, D. \& Fibiger, H. C. Sexual activity increases dopamine transmission in the nucleus accumbens and striatum of female rats. Brain Res 693, 21-30, doi:10.1016/0006-8993(95)00679-k (1995).

35 Becker, J. B., Rudick, C. N. \& Jenkins, W. J. The role of dopamine in the nucleus accumbens and striatum during sexual behavior in the female rat. $J$ Neurosci 21, 32363241 (2001).

36 Jenkins, W. J. \& Becker, J. B. Dynamic increases in dopamine during paced copulation in the female rat. Eur J Neurosci 18, 1997-2001, doi:10.1046/j.1460-9568.2003.02923.x (2003).

37 Sun, F. et al. A Genetically Encoded Fluorescent Sensor Enables Rapid and Specific Detection of Dopamine in Flies, Fish, and Mice. Cell 174, 481-496 e419, doi:10.1016/j.cell.2018.06.042 (2018).

38 Sharma, N. et al. The emergence of transcriptional identity in somatosensory neurons. Nature 577, 392-398, doi:10.1038/s41586-019-1900-1 (2020).

39 Maksimovic, S. et al. Epidermal Merkel cells are mechanosensory cells that tune mammalian touch receptors. Nature 509, 617-621, doi:10.1038/nature13250 (2014).

40 Neubarth, N. L. et al. Meissner corpuscles and their spatially intermingled afferents underlie gentle touch perception. Science 368, 1330-+, doi:ARTN eabb2751

10.1126/science.abb2751 (2020).

41 Rodgers, C. C. et al. Sensorimotor strategies and neuronal representations for shape discrimination. Neuron 109, 2308-+, doi:10.1016/j.neuron.2021.05.019 (2021).

42 Severson, K. S. et al. Active Touch and Self-Motion Encoding by Merkel Cell-Associated Afferents. Neuron 94, 666-+, doi:10.1016/j.neuron.2017.03.045 (2017).

43 Seal, R. P. et al. Injury-induced mechanical hypersensitivity requires C-low threshold mechanoreceptors. Nature 462, 651-655, doi:10.1038/nature08505 (2009).

44 Seal, R. P. et al. Injury-induced mechanical hypersensitivity requires C-low threshold mechanoreceptors. Nature 462, 651-655, doi:10.1038/nature08505 (2009).

45 Francois, A. et al. The Low-Threshold Calcium Channel Cav3.2 Determines LowThreshold Mechanoreceptor Function. Cell Rep 10, 370-382, doi:10.1016/j.celrep.2014.12.042 (2015).

46 Urien, L. et al. Genetic ablation of GINIP-expressing primary sensory neurons strongly impairs Formalin-evoked pain. Sci Rep 7, 43493, doi:10.1038/srep43493 (2017).

47 Bohic, M. et al. Loss of bhlha9 Impairs Thermotaxis and Formalin-Evoked Pain in a Sexually Dimorphic Manner. Cell Rep 30, 602-610 e606, doi:10.1016/j.celrep.2019.12.041 (2020).

$48 \mathrm{Li}, \mathrm{L}$. \& Ginty, D. D. The structure and organization of lanceolate mechanosensory complexes at mouse hair follicles. Elife 3, e01901, doi:10.7554/eLife.01901 (2014). 
49 Kuehn, E. D., Meltzer, S., Abraira, V. E., Ho, C. Y. \& Ginty, D. D. Tiling and somatotopic alignment of mammalian low-threshold mechanoreceptors. Proc Natl Acad Sci U S A 116, 9168-9177, doi:10.1073/pnas.1901378116 (2019).

50 Morales, M. \& Margolis, E. B. Ventral tegmental area: cellular heterogeneity, connectivity and behaviour. Nat Rev Neurosci 18, 73-85, doi:10.1038/nrn.2016.165 (2017).

51 Lammel, S., Ion, D. I., Roeper, J. \& Malenka, R. C. Projection-specific modulation of dopamine neuron synapses by aversive and rewarding stimuli. Neuron 70, 855-862, doi:10.1016/j.neuron.2011.03.025 (2011).

52 Cora, M. C., Kooistra, L. \& Travlos, G. Vaginal Cytology of the Laboratory Rat and Mouse: Review and Criteria for the Staging of the Estrous Cycle Using Stained Vaginal Smears. Toxicol Pathol 43, 776-793, doi:10.1177/0192623315570339 (2015).

53 Burkett, J. P. et al. Oxytocin-dependent consolation behavior in rodents. Science 351, 375-378, doi:10.1126/science.aac4785 (2016).

$54 \mathrm{Lu}, \mathrm{Y}$. F. et al. Social interaction with a cagemate in pain increases allogrooming and induces pain hypersensitivity in the observer rats. Neurosci Lett 662, 385-388, doi:10.1016/j.neulet.2017.10.063 (2018).

55 Bruno, C. A. et al. pMAT: An open-source software suite for the analysis of fiber photometry data. Pharmacol Biochem Behav 201, 173093, doi:10.1016/j.pbb.2020.173093 (2021). 